\title{
GENERALISED KAWADA-SATAKE METHOD FOR MACKEY FUNCTORS IN CLASS FIELD THEORY
}

\author{
IVAN B. FESENKO, SERGEI V. VOSTOKOV, SEOK HO YOON
}

\begin{abstract}
We propose and study a generalised Kawada-Satake method for Mackey functors in the class field theory of positive characteristic. The root of this method is in the use of explicit pairings, such as the ArtinSchreier-Witt pairing, for groups describing abelian extensions. We separate and simplify the algebraic component of the method and discuss a relation between the existence theorem in class field theory and topological reflexivity with respect to the explicit pairing. We apply this method to derive higher local class field theory of positive characteristic, using advanced properties of topological Milnor K-groups of such fields.
\end{abstract}

\section{CONTENTS}

1. Introduction 1

2. Classical Witt theory 5

3. Generalised Kawada-Satake class field theory mechanism for Mackey functors 7

3.1. Pairable sequences of groups 8

3.2. Special Mackey functors 9

3.3. Mackey functors for fields of positive characteristic 10

3.4. Class field theory mechanism 13

3.5. Remarks and Examples 14

3.6. Class field theories of one-dimensional fields of positive characteristic 15

4. Class field theory of higher local fields of positive characteristic 18

4.1. Higher local fields of positive characteristic 18

4.2. Topology on higher local fields 19

4.3. Milnor and topological Milnor $K$-groups of higher local fields 21

4.4. Generalised Artin-Schreier-Witt pairing 22

4.5. The group $\Lambda_{n}(F)$ and the norm map 24

4.6. Higher local class field theory in positive characteristic 25

$\begin{array}{ll}\text { References } & 27\end{array}$

\section{INTRODUCTION}

Generations of number theorists were interested to deduce main results of various types of class field theories from as few axioms as possible. Starting with Artin and Tate, such axioms were often called class formation. Let $G$ be a topological pro-finite group. Let $A$ be a $G$-module. For any open subgroup $H$ of $G$, denote by $A_{H}$ the submodule of $H$-fixed elements of $A$. For any open subgroup $K$ of any open subgroup $H$ of $G$ we have the morphism $i_{H / K}: A_{H} \rightarrow A_{K}$ induced by the embedding and the morphism $\sigma^{*}: A_{H} \rightarrow A_{\sigma H \sigma^{-1}}$ induced by the 
multiplication by $\sigma \in G$. We also have the well defined norm morphism $N_{H / K}: A_{K} \rightarrow A_{H}$ given by $\alpha \mapsto\left(\sum \sigma\right) \alpha$ where $\sigma$ runs through a set of representatives of cosets of $H$ modulo $K$. Following the Artin-Tate approach, the axioms of class formation typically included the following two: $H^{2}\left(H / K, A_{K}\right)$ is cyclic of order $|H: K|$ when $K$ is a normal open subgroup of $H, H^{1}\left(H / K, A_{K}\right)=0$ when $K$ is a normal open subgroup of $H$ of prime index, see e.g. [1].

From the axioms one derives the existence of the reciprocity homomorphism $\Phi_{H}: A_{H} \longrightarrow H^{\mathrm{ab}}$ for every open subgroup $H$ of $G$. One proves that for an open subgroup $K$ of $H$ this reciprocity homomorphism induces an isomorphism $A_{H} / N_{H / K} A_{K} \simeq(H / K)^{\mathrm{ab}}$. The reciprocity homomorphism $\Phi_{H}$ satisfies a number of natural compatibility properties with respect to the above morphisms. On $A_{H}$ one can introduce a translation invariant topology in which open neighbourhoods of zero are $N_{H / K} A_{K}$ where $K$ runs through all open subgroups $K$ of $H$, call it the normic topology. One proves that for every open subgroup $H$ of $G$ there is a bijective correspondence between open subgroups $K$ of $H$ with abelian factors $H / K$ and open normic subgroups of $A_{H}$, see e.g. [31].

We call the algorithmic deduction of main theorems of class field theory from class formation (or class field theory) axioms a class field theory mechanism. Thus, class field theory may be viewed as consisting of two different from each other parts: (a) a chosen class field theory mechanism, (b) checking axioms of the chosen class field theory mechanism for a specific type of groups $G$, typically Galois groups of a specific type of fields.

If $A_{H}$ are endowed with some original topologies compatible with various morphisms, including $\sigma^{*}, i$ and $N$, then, following the 'class fields' task, one is interested to describe the normic topology in terms of the original topology of $A_{H}$, this is called the existence theorem. For the purposes of class field theories, one usually takes $G$ as the Galois group of a Galois field extension $l / k$. In particular, the existence theorem describes abelian subextensions of $k$ inside $l$ in terms of open subgroups of $A_{G}$. A natural question is whether the original topology is equivalent to the normic topology. The answer is positive for classical (one-dimensional) global fields and their completions, but negative already for complete discrete valuation fields with an infinite quasifinite residue field of positive characteristic (a perfect field whose absolute Galois group is isomorphic to $\hat{\mathbb{Z}}$ ), see e.g. [15, Ch.5 sect.3].

Many presentations of class field theories have their class field theory mechanism written down in a purely group-theoretical and Galois module-theoretical way, without using ring structures. From the point of view of mono-anabelian geometry of Mochizuki [30], such group-theoretical tools can be named mono-anabelian, since they do not depend on the ring structure but only on the topological group structure of objects involved in class field theory. On the other hand, to establish class field theory axioms, i.e. part (b) of class field theory, typically requires the use of ring structure of the fields involved.

If $G$ is the absolute Galois group of a complete discrete valuation field $F$ with a finite residue field (so $F$ is often called a local field) and $A$ is the multiplicative group of a separable closure of $F$, using the ring structure of $F$ one checks that the class formation axioms are satisfied. Therefore via the class field theory mechanism one gets the local reciprocity homomorphism $\Phi_{L}: L^{\times} \longrightarrow G_{L}^{\mathrm{ab}}$ for every finite separable extension $L$ of $F$. It is known that local class field theory (i.e. the local reciprocity homomorphism) is not of mono-anabelian nature, i.e. it does depend on the ring structure of local fields, see e.g. [30]. While the class field theory mechanism is mono-anabelian, the verification of the axioms cannot be produced in a mono-anabelian way.

If $G$ is the absolute Galois group of a global field $F$ and $A$ is the direct limit of idele class groups $C_{L}$ of finite separable extensions $L$ of $F$ in a fixed separable closure of $F$, using the ring structure of $F$ one checks that the axioms for $A$ are satisfied and one derives the global reciprocity homomorphism $\Phi_{L}: C_{L} \longrightarrow G_{L}^{\mathrm{ab}}$. Typically, one uses local class field theory to derive global class field theory. For a global field $L$ the reciprocity homomorphism $\Phi_{L}$ is the product of all local homomorphisms $\Phi_{L_{v}}$ for all completions $L_{v}$ of $L$ with respect to 
all places $v$ of $L$, with appropriately defined archimedean reciprocity maps for archimedean places. One has a natural topology on $A$ in these two cases and one proves the existence theorem.

Many best known approaches to the class field theory mechanism use a computation of the Brauer group or the second Galois cohomology group. Hazewinkel and Iwasawa suggested cohomology free approaches to local class field theory, see e.g. [15, Ch.IV]. Neukirch suggested a very explicit and essentially cohomologyfree (and the Brauer group-free) method for the class field theory mechanism for local and global class field theories. His method emphasises and generalises the role of a distinguished $\hat{\mathbb{Z}}$-quotient of $G$ that is available in the local and global cases, [31], [32]. In the case of local fields the distinguished $\hat{\mathbb{Z}}$-quotient is the extension originating from the residue fields level, it is the maximal unramified extension of the local field. In the case of rational numbers the distinguished $\hat{\mathbb{Z}}$-quotient corresponds to the unique $\hat{\mathbb{Z}}$-subextension of the maximal abelian extension of the field of rational numbers. In the case of global fields of positive characteristic the distinguished $\hat{\mathbb{Z}}$-quotient corresponds to the maximal constant extension of the field — this was not included in [31], [32], for an application of the Neukirch method to class field theory of global fields of positive characteristic see [42, sect.3.4].

A new axiom in the Neukirch class field theory mechanism asks for the existence of (i) a normal closed subgroup $G_{0}$ of $G$ such $G / G_{0}$ is isomorphism to $\hat{\mathbb{Z}}$ and (ii) a group homomorphism $v: A_{G} \longrightarrow \hat{\mathbb{Z}}$ whose image is $\mathbb{Z}$ or $\hat{\mathbb{Z}}$, (iii) the compatibility: $v\left(N_{G / H} A_{H}\right)=\left|G: H G_{0}\right| v\left(A_{G}\right)$ for all open subgroups $H$ of $G$. Modulo the new axiom, i.e. taking into account the role of the distinguished $\hat{\mathbb{Z}}$-quotient of $G$, other Neukirch's axioms are simpler than the Artin-Tate class formation axioms: all one needs to know is that for normal open subgroups $K$ of $H$ such that $H / K$ is cyclic the cokernel of $N_{H / K}$ is a cyclic group of order $|H / K|$ and the kernel of $N_{H / K}$ is the submodule $\left\{(\sigma-1) \alpha: \alpha \in A_{K}, \sigma \in H\right\}$, [31]. The reciprocity homomorphism is deduced from the Neukirch axioms in a purely group-theoretical argument.

The Neukirch method clarified the place of cohomological computations in class field theory: there are class field theory mechanisms which do not use them and checking that their axioms hold for specific fields does not use cohomological computations either. Thus, class field theory can be derived without the knowledge of the structure of the second cohomology groups such as the Brauer group. For example, two theories of Mochizuki, his mono-anabelian geometry [30] and inter-universal Teichmüller theory, use Kummer theory (which has many structural analogies to Witt theory in positive characteristic) and the computation of the Brauer group (or appropriate $H^{2}$ ) of the local fields, but they do not use any local class field theory mechanism.

The paper [26] of Kawada and Satake derived the p-primary part of class field theory of local and global fields of positive characteristic $p$, using Witt theory that describes the $p$-part of the maximal abelian extension of a field in terms of the direct limit of the quotients of truncated Witt vectors modulo the operator $\mathbf{F}-$ id. This method was extended to class field theory of local fields of positive characteristic with a $p$-quasi-finite residue field (i.e. it is perfect of characteristic $p$ and the Galois group of its maximal separable $p$-extension is isomorphic to the group $\mathbb{Z}_{p}$ ) in [35].

There are many variations of class field theory mechanisms and class field theory axioms, to make the theory work for larger and larger classes of (one-dimensional) local and global fields, see, e.g. [25], [26]. Finding new examples of fields for which there is a kind of class field theory but the existing class field mechanism axioms are too restrictive to be applicable seems to be a never ending process. More recent examples include higher class field theory [4], p-class field theory [5], [6], general class field theory [7] (where, unusually, there is no induction of the degree of extension), non-commutative local class field theories [8], [9], [16], [17], [18].

Together with the representation-theoretical Langlands program and anabelian geometry, higher class field theory is one of the main generalisations of class field theory. It studies abelian extensions of higher dimensional 
local and global fields. When the dimension is one, this is just the classical class field theory. Higher class field theory is a very non-trivial generalisation of class field theory, since various properties of objects used in the classical theory do not hold any longer. For $n$-dimensional fields, instead of the multiplicative group of the field or the idele class group one uses for $A_{H}$ the Milnor $K_{n}$-groups or certain quotients of so called topological Milnor $K_{n}$-groups for local theories and their adelic versions for local-global and global theories. Note that the Galois descent (i.e. $A_{H} \rightarrow A_{K}^{G(H / K)}$ is an isomorphism), which plays the fundamental role in dimension one, does not typically hold for abelian groups $A_{H}$ in higher class field theory. Indeed, for a finite Galois extension $L / F$ of $n$-dimensional fields, the Galois invariant elements of $K_{n}(L)$ are not generally isomorphic to $K_{n}(F)$ and the map $K_{n}(F) \rightarrow K_{n}(L)$ induced by the embedding $F \rightarrow L$ is not injective in general.

Kato generalised the classical (one-dimensional) cohomological approach, [22]. In his approach the Brauer group $H^{2}(G, \mu)$ is replaced with the group $H^{n+1}\left(G, \mu^{\otimes n}\right)$. Unlike the Brauer group, these groups for $n>1$ do not play the same central role in algebra, but they are still important. While [22] were not the easiest papers to follow, a certain simplification of [22] was included in [23]. The most difficult part of [22] is perhaps the $p$-primary part in positive characteristic $p$. Kato's local theory was further extended to higher global class field theory in his joint work with Saito [24].

Later, Parshin published the papers [33], [34] on higher local class field theory in positive characteristic using a generalisation of the Kawada-Satake method and working with Milnor $K$-groups endowed with a certain topology and a newly defined norm map between them. Unlike the Kato approach [22], the $p$-primary part of the Parshin approach looked relatively easy. Unfortunately, the definition of the topology on the multiplicative group and Milnor $K$-groups of $n$-dimensional local fields was incorrect for $n>2$, the study of properties of the new norm map contained gaps, and the existence theorem was incorrect.

At the same time, Fesenko constructed two-dimensional local class field theory in [3] generalising the Weil method developed of [39, sect. 1 Ch. 12] and using some of Kato's results. The papers [4] developed higher class field theory via a generalised version of the Neukirch method [31], by working with certain topological quotients $K_{n}^{\text {top }}$ of the Milnor $K$-groups of the fields and checking all the relevant axioms. Note that the classical (one-dimensional) class formation axioms and the usual Neukirch's axioms do not apply in higher class field theory, in the absence of Galois descent for topological Milnor $K$-groups. The papers [4] demonstrated the power of topological considerations for class field theory. In particular, they contained the first published proof of the existence theorem for all higher local fields. An extension of the generalised Neukirch method to twodimensional global class field theory was briefly stated in [12, sect.2].

In a conceptually different direction, Koya found a generalised class formation approach to higher class field theory using bounded complexes of Galois modules and their modified hyper-cohomology groups instead of respectively Galois modules and their modified (Tate) cohomology groups, [28]. His approach uses the complex $\mathbb{Z}(n)$ and at that time he could apply his method to two-dimensional local fields only. Spieß found for all $n$ a substitute of the complex $\mathbb{Z}(n)$ that is suitable for Koya's approach to be applied to all higher local fields, [36]. A positive feature of this approach is its fit with central developments in motivic cohomology such as the use of the Suslin-Voevodsky complex $\mathbb{Z}(n)$, its drawbacks is its relative lack of explicitness.

See [13] for a recent analysis of class field theory types and their generalisations.

We now describe the contents of this paper. After recalling basic facts about Witt theory in the second section, the third section presents a version of generalised Kawada-Satake method for class field theory mechanism. We offer a new simplified version and its generalisation. It includes the use of an algebraic component dealing with pairable sequences of abelian groups. The generalisation involves Mackey functors from the category of left $G$-sets. Mackey functors were already used in [4] and [6], without explicitly naming them. We formulate key 
properties/axioms which can be viewed as general class formation axioms for the generalised Kawada-Satake method and Mackey functors. These axioms deal with more general objects than Galois modules and they do not include any of the standard class formation axioms or their modifications, such as the information on the index of the norm group and the kernel of the norm map. In section three we prove that the axiomatic properties imply all main theorems of the $p$-primary part of class field theory in characteristic $p$. In this generalised Kawada-Satake approach the reflexivity of closed subgroups of of finite index is closely related to the existence theorem. This provides a topological duality perspective on the meaning of the existence theorem.

In the fourth section we check that the axioms of the generalised Kawada-Satake method are satisfied for higher local fields of positive characteristic and their topological Milnor $K$-groups. We work with sequentially saturated topologies on higher local fields and their Milnor $K$-groups. In particular, errors in [33], [34] are discussed and corrected in Remarks 4.8, 4.17. A very short sketch of how to correct [33], [34] was included in [11], but in this paper we go significantly further, also including many more details. In the study of properties of topological Milnor $K$-groups and the norm map on them, a key theorem from [10] about an algebraic description of the intersection of open neighbourhoods of zero in the topological Milnor $K$-groups finds its application. After checking that all the class field theory axioms of the third section are satisfied for higher local fields, we obtain a new presentation of explicit class field theory of higher local fields of positive characteristic via the generalised Kawada-Satake method of this paper.

Work on this paper was partially supported by EPSRC programme grant 'Symmetries and Correspondences'EP/M024830.

In this paper all characters of groups that are endowed with a topology will be continuous characters. For a group endowed a translation invariant topology, from section 3 on we use the notation $A^{\odot}$ for the group of its (continuous) characters endowed with the compact-to-open (compact-open) topology. It will be convenient for a group $A$ endowed with the discrete topology to denote its group of characters also by $A^{\circ}$.

The groups $\mathbb{Z}, \mathbb{Z} / p^{m} \mathbb{Z}, p^{-m} \mathbb{Z} / \mathbb{Z}, \mathbb{Q}_{p} / \mathbb{Z}_{p}$ will be endowed with the discrete topology.

For a Galois field extension $L / F$ its Galois group is denoted $G(L / F)$.

In this paper we will work with fields of positive characteristic $p$.

\section{Classical Witt theory}

For the convenience of the reader we include in this section a brief summary of classical Witt theory.

Let $k$ be a field of characteristic $p>0$. Fix a separable closure $k^{\text {sep }}$ of $k$. Denote by $k^{\text {sep, } p}$ the maximal $p$-extension of $k$ inside $k^{\mathrm{sep}}$, put $G^{\mathrm{sep}, p}(k):=G\left(k^{\mathrm{sep}, p} / k\right)$. Denote by $k^{\mathrm{ab}, p}$ its maximal abelian subextension, put $G^{\mathrm{ab}, p}(k):=G\left(k^{\mathrm{ab}, p} / k\right)$.

Let $W(k)$ be the $\left(p\right.$-)Witt ring of $k$, so $W(k)=\left\{w=\left(w_{0}, w_{1}, \ldots\right): w_{i} \in k\right\}$, with appropriate ring structure, see e.g. [15, Ch.I sect.8]. It is the inverse limit of the rings of truncated Witt vectors $W_{m}(k)=\left\{\left(w_{0}, \ldots, w_{m-1}\right)\right.$ : $\left.w_{i} \in k\right\}, m \geqslant 1$, with appropriate ring structure, with respect to the surjective homomorphisms

$$
p_{m}: W_{m}(k) \rightarrow W_{m-1}(k),\left(w_{0}, \ldots, w_{m-1}\right) \mapsto\left(w_{0}, \ldots, w_{m-2}\right) .
$$

Let $\mathbf{V}, \mathbf{F}: W(k) \rightarrow W(k)$ be the Verschiebung and the Frobenius homomorphisms

$$
\mathbf{V}\left(\left(w_{0}, w_{1}, \ldots\right)\right)=\left(0, w_{0}, w_{1}, \ldots\right), \quad \mathbf{F}\left(\left(w_{0}, w_{1}, \ldots\right)\right)=\left(w_{0}^{p}, w_{1}^{p}, \ldots\right), \quad \mathbf{V F}=\mathbf{F V}=p
$$


As usual, denote $\wp=\mathbf{F}-$ id, so $\wp(w)=\mathbf{F}(w)-w$.

In this paper we will only use the additive group structure of $W(k)$. The groups $W_{m}(k)=W(k) / \mathbf{V}^{m} W(k)$ are of exponent $p^{m}$. The translation invariant topology of $W(k)$ is defined by taking $\mathbf{V}^{r} W(k)$ as the fundamental system of neighbourhoods of the identity element. The additive group $W(k)$ is a topological group. The group $W_{m}(k)$ is endowed with the induced topology, with respect to which it is a discrete group; in particular, the additive group of $k$ is discrete.

Recall the classical

Proposition 2.1. Let ${ }_{m} k / k$ be the maximal abelian extension of exponent $p^{m}$, i.e. the compositum of all cyclic extensions of degree $p^{m}$ of $k$.

The Witt pairing

$$
G\left({ }_{m} k / k\right) \times W_{m}(k) / \wp\left(W_{m}(k)\right) \longrightarrow W_{m}\left(\mathbb{F}_{p}\right) / \wp\left(W_{m}\left(\mathbb{F}_{p}\right)\right) \simeq \mathbb{Z} / p^{m} \mathbb{Z}, \quad(g, w) \mapsto g w^{\prime}-w^{\prime}, \wp\left(w^{\prime}\right)=w
$$

is continuous in each variable and perfect. Hence in the category of topological groups $W_{m}(k) / \wp\left(W_{m}(k)\right)$ is isomorphic to $\operatorname{Hom}_{\text {cont }}\left(G^{\mathrm{ab}, p}(k), \mathbb{Z} / p^{m} \mathbb{Z}\right)=\operatorname{Hom}_{\text {cont }}\left(G\left({ }_{m} k / k\right), \mathbb{Z} / p^{m} \mathbb{Z}\right)=G\left({ }_{m} k / k\right)^{\circ}$ and $G\left({ }_{m} k / k\right)$ is isomorphic to $\mathrm{Hom}_{\text {cont }}\left(W_{m}(k) / \wp\left(W_{m}(k)\right), \mathbb{Z} / p^{m} \mathbb{Z}\right)=\left(W_{m}(k) / \wp\left(W_{m}(k)\right)\right)^{\circ}$.

There is a bijective correspondence between subgroups $V$ in $W_{m}(k)$ that contain $\wp\left(W_{m}(k)\right)$ and abelian extensions $l / k$ of exponent $p^{i}, i \leqslant m: V \leftrightarrow l=k\left(\wp^{-1}(V)\right)$, where $k\left(\wp^{-1}(V)\right)$ is the compositum of the fields $k\left(w_{0}, \ldots, w_{m-1}\right)$ such that $\wp\left(w_{0}, \ldots, w_{m-1}\right) \in V$.

For more detail see [40] or [29, Ch.VIII exercises].

Remark 2.2. Many arguments in Witt theory are parallel to those in Kummer theory, however, the latter requires roots of unity in the ground field while the former is free from any such restriction in characteristic $p$.

Remark 2.3. Witt theory is an abelian extension of Artin-Schreier theory. A non-abelian extension of ArtinSchreier theory was developed by Inaba [19], [20]. Inaba theory seems to be virtually unknown to mathematicians working with non-abelian extensions of fields of positive characteristic. Inaba theory should find various applications to theories in positive characteristic, including aspects of the Langlands program.

The group $G^{a b, p}(k)$ is the inverse limit of the groups $G\left({ }_{m} k / k\right)$. Hence it is isomorphic to the group of characters of the direct limit of groups $W_{m}(k) / \wp\left(W_{m}(k)\right)$ with respect to appropriate morphisms, which we now describe.

Definition 2.4. Define

$\mathscr{W}(k)=W(k) / \wp(W(k)) \otimes \mathbb{Q}_{p} / \mathbb{Z}_{p}, \quad \mathscr{W}_{m}(k)=W_{m}(k) / \wp(W(k)) \otimes p^{-m} \mathbb{Z} / \mathbb{Z}=W(k) / \wp(W(k)) \otimes p^{-m} \mathbb{Z} / \mathbb{Z}$.

Then $\mathscr{W}_{m}(k)$ is the subgroup of $\mathscr{W}(k)$ of its elements of order $p^{i}, i \leqslant m$.

If $\operatorname{dim}_{\mathbb{F}_{p}} k / \wp(k)=\kappa$, then $\mathscr{W}(k) \simeq\left(\mathbb{Q}_{p} / \mathbb{Z}_{p}\right)^{\oplus \kappa}$.

The group $\mathscr{W}(k)$ is the direct limit of $\mathscr{W}_{m}(k)$ with respect to the canonical injective morphisms $i_{m}: \mathscr{W}_{m}(k) \rightarrow$ $\mathscr{W}_{m+1}(k)$. Define its topology as the direct limit of the discrete topologies of $\mathscr{W}_{m}(k)$, so the topology of $\mathscr{W}(k)$ is discrete.

Remark 2.5. In the next sections $W_{m}(k), \mathscr{W}(k)=\underline{\lim _{\longrightarrow}} \mathscr{W}_{m}(k)$ will be endowed with new topologies depending on the topology of $k$. 
We have isomorphisms

$$
\begin{aligned}
\phi_{m}: & W_{m}(k) / \wp\left(W_{m}(k)\right) \simeq \mathscr{W}_{m}(k), \\
& \quad\left(w_{0}, w_{1}, \ldots, w_{m-1}\right)+\wp\left(W_{m}(k)\right) \mapsto\left(\left(w_{0}, w_{1}, \ldots, w_{m-1}, 0, \ldots\right)+\wp(W(k)) \otimes 1 / p^{m} .\right.
\end{aligned}
$$

Define

$$
\psi_{m}: W_{m}(k) / \wp\left(W_{m}(k)\right) \rightarrow W_{m+1}(k) / \wp\left(W_{m+1}(k)\right), \quad \psi_{m}=\phi_{m+1}^{-1} \circ i_{m} \circ \phi_{m} .
$$

One immediately checks that the homomorphism $\psi_{m}$ is induced by $\mathbf{V}$, it sends $\left(w_{0}, \ldots, w_{m-1}\right)+\wp\left(W_{m}(k)\right)$ to $\left(0, w_{0}^{p}, \ldots, w_{m-1}^{p}\right)+\wp\left(W_{m+1}(k)\right)=\mathbf{V}\left(w_{0}, \ldots, w_{m-1}\right)+\wp\left(W_{m+1}(k)\right)$.

The commutative diagram

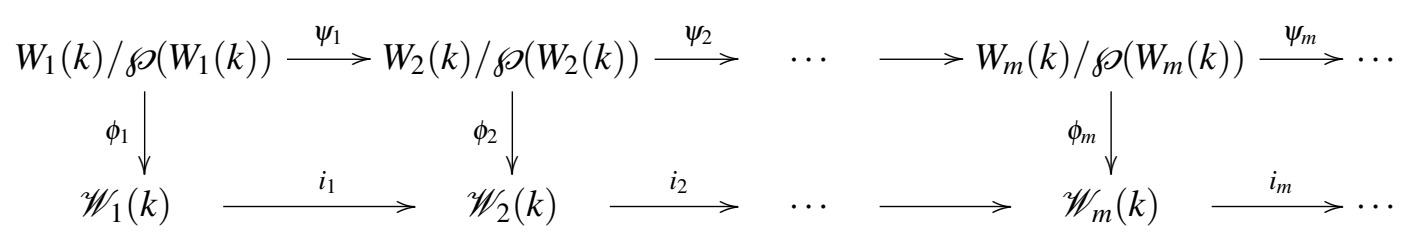

shows that $\mathscr{W}(k)$ is isomorphic to the direct limit of $W_{m}(k) / \wp\left(W_{m}(k)\right)$ with respect to $\psi_{m}=\mathbf{V}$.

Using Proposition 2.1, one immediately gets

Proposition 2.6. In the category of topological groups, Witt theory implies isomorphisms of discrete and compact groups

$$
\mathfrak{v}_{k}: \mathscr{W}(k) \longrightarrow G^{a b, p}(k)^{\circ}, \quad \mathfrak{w}_{k}: G^{a b, p}(k) \longrightarrow \mathscr{W}(k)^{\circ} .
$$

For other details about Witt rings, see e.g. [35, sect.2].

The Galois action on fields commutes with the functors $W_{m}, W, \mathscr{W}$. The groups $W_{m}(l), \mathscr{W}(l)$ coincide with $G\left(k^{\mathrm{sep}, p} / l\right)$-invariant elements of $W_{m}\left(k^{\mathrm{sep}, p}\right), \mathscr{W}\left(k^{\mathrm{sep}, p}\right)$; the group $\mathscr{W}(l)^{\circ}$ coincides with $G\left(k^{\mathrm{sep}, p} / l\right)$-invariant elements of $\mathscr{W}\left(k^{\mathrm{sep}, p}\right)^{\circ}$.

Remark 2.7. We can ask if we are in the situation of class formation given by the pro- $p$-group $G=G\left(k^{\mathrm{sep}, p} / k\right)$ and the $G$-module $A=\mathscr{W}\left(k^{\text {sep }, p}\right)^{\circ}$. It is straightforward to check that the answer is positive: (1) for any normal open subgroup $K$ of an open subgroup $H$ of $G$ the group $H^{2}\left(H / K, A_{K}\right)$ is cyclic of order $|H / K|$, (2) $H^{1}\left(H / K, A_{K}\right)=0$; for details see e.g. [27]. Thus, the isomorphism $A_{k}=\mathscr{W}(k)^{\circ} \longrightarrow G^{a b, p}(k)$ is the reciprocity isomorphism with respect to this class formation. For the existence theorem in this class field theory see [27, sect.1].

\section{Generalised KAWADA-SATAKE ClASS FIELD THEORY MECHANISM FOR MACKEy FUNCTORS}

We now describe a generalisation of the Kawada-Satake method for class field theory mechanisms in positive characteristic, using Mackey functors and Witt theory. As far as class field theory is concerned, Mackey functors were already used in [4] and [6], without explicitly naming them, and they are mentioned in [32, Ch.IV sect.6 Exercises]. We start with the notion of pairable sequences of abelian groups. 


\subsection{Pairable sequences of groups.}

Definition 3.1. (pre-pairable sequence of groups) A sequence $A_{m}, m \geqslant 1$, of abelian groups is called prepairable if $p^{m} A_{m}=0$ for all $m \geqslant 1$, and for all $m>1$ there are homomorphisms $i_{m}: A_{m-1} \rightarrow A_{m}$ and surjective homomorphisms $p_{m}: A_{m} \rightarrow A_{m-1}$ such that $\operatorname{ker}\left(p_{m}\right)=p^{m-1} A_{m}$,

$$
p_{m} \circ i_{m}=p \operatorname{id}_{A_{m-1}}, \quad i_{m} \circ p_{m}=p \operatorname{id}_{A_{m}} .
$$

The composite $p_{r+1} \circ \cdots \circ p_{m}$ induces the isomorphism $A_{m} / p^{r} A_{m} \simeq A_{r}$ for $r<m$.

Examples 3.2. (a) for a semiabelian variety $A$ over an algebraically closed field $k$, let $A_{m}=\left\{x \in A(k): p^{m} x=0\right\}$, let morphisms $i_{m}$ be embeddings and morphisms $p_{m}$ be multiplication by $p$;

(b) let $A_{m}=\mu_{p^{m}}^{\otimes r}$ with obvious morphisms;

(c) for an abelian group $A$, let $A_{m}=A / p^{m} A$, let $i_{m}$ be multiplication by $p$ and $p_{m}$ be the natural surjections;

(d) $A_{m}=W_{m}(k)$ as in the previous section, and let morphisms $i_{m}$ be $\mathbf{V}$ and morphisms $p_{m}$ be $p_{m}$;

(e) $A_{m}=W_{m}(k) / \wp\left(W_{m}(k)\right)$ as in the previous section, and let morphisms $i_{m}$ be induced by $\mathbf{V}$ and morphisms $p_{m}$ be $p_{m}$.

Note that the morphisms $i_{m}$ are injective in (a), (b),(d),(e), but not in general in (c).

Definition 3.3. (two pairable sequences of groups) Let $A_{m}, m \geqslant 1$, be a pre-pairable sequence of groups and let $B_{m}, m \geqslant 1$, be a pre-pairable sequence of groups.

Suppose that all of these groups are endowed with topologies (not necessarily making them topological groups) such that the structure morphisms $i_{m}, p_{m}$ are continuous and closed, so for every closed subset its image and its preimage are closed. Suppose that for all $r<m$ the isomorphisms $A_{m} / p^{r} A_{m} \simeq A_{r}, B_{m} / p^{r} B_{m} \simeq B_{r}$, induced by $p_{j}$, are homeomorphisms.

The two sequences $A_{m}, B_{m}$ are called pairable if for all $m \geqslant 1$ there are bi-linear pairings $():, A_{m} \times B_{m} \rightarrow$ $\mathbb{Z} / p^{m} \mathbb{Z}$ continuous in each variable and satisfying

$$
\left(p_{m} a, b\right)_{m-1}=p_{m}\left(a, i_{m} b\right)_{m}, \quad\left(a, p_{m} b\right)_{m-1}=p_{m}\left(i_{m} a, b\right)_{m},
$$

where we also use the notation $p_{m}$ for the pre-pairable sequence of $\mathbb{Z} / p^{m} \mathbb{Z}$ as in Example (c).

There are induced homomorphisms $\zeta_{m}: A_{m} \rightarrow \operatorname{Hom}_{\text {cont }}\left(B_{m}, \mathbb{Z} / p^{m} \mathbb{Z}\right), \xi_{m}: B_{m} \rightarrow \operatorname{Hom}_{\text {cont }}\left(A_{m}, \mathbb{Z} / p^{m} \mathbb{Z}\right)$. The morphisms $i_{m}, p_{m}$ for $A_{m}, B_{m}$ and $\mathbb{Z} / p^{m} \mathbb{Z}$ induce morphisms $i_{m}^{*}, p_{m}^{*}$ on Hom-groups. We have the commutative diagrams
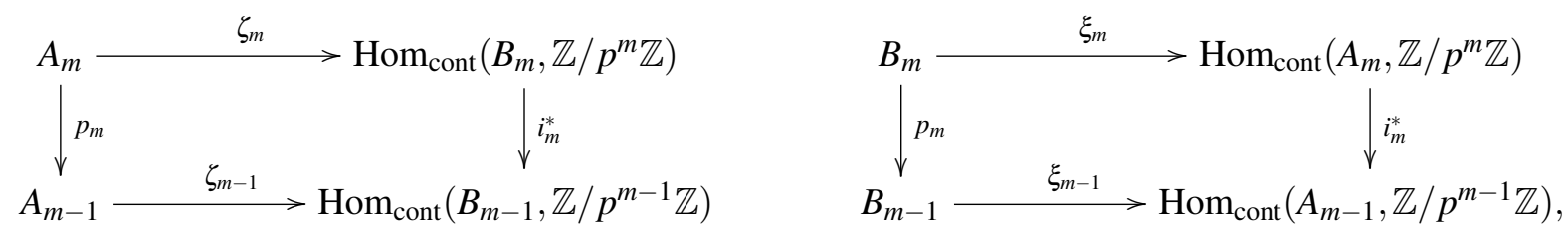

where $i_{m}^{*}(\chi)=p_{m} \circ \chi \circ i_{m}$. Note that the other similar diagram, with $i_{m}$ on the left and $p_{m}^{*}$ on the right, is not commutative.

Definition 3.4. Recall that the pairing $(,)_{m}$ is called non-degenerate if $\zeta_{m}$ and $\xi_{m}$ are injective. The pairing $(,)_{m}$ is called perfect if $\zeta_{m}$ and $\xi_{m}$ are isomorphisms, both algebraically and topologically. We will call the pairing $(,)_{m}$ algebraically perfect if $\zeta_{m}$ and $\xi_{m}$ are group isomorphisms, without any restriction on their topological properties. 
Lemma 3.5. Let $A_{m}$ and $B_{m}$ be pairable sequences of groups. If the pairing $(,)_{1}$ is non-degenerate (resp. non-degenerate on the left) then the pairings $(,)_{m}$ are non-degenerate (resp. non-degenerate on the left) for all $m \geqslant 1$. If the pairing $(,)_{1}$ is algebraically perfect (resp. perfect on the left) then the pairings $(,)_{m}$ are algebraically perfect (resp. perfect on the left) for all $m \geqslant 1$.

Proof. Assume that all $(,)_{r}$, for $r<m$, are non-degenerate. Let $\zeta_{m}(a)=0$, then, due to the induction assumption and the commutative diagram before this lemma, $p_{m}(a)=0$, hence $a=p^{m-1} a^{\prime}$. Then $0=\left(p^{m-1} a^{\prime}, b\right)_{m}=$ $\left(i_{m} \circ \ldots i_{2} \circ p_{2} \circ \ldots p_{m}\left(a^{\prime}\right), b\right)_{m}=\left(p_{2} \circ \ldots p_{m}\left(a^{\prime}\right), p_{2} \circ \ldots p_{m}(b)\right)_{1}$ for all $b \in B_{m}$, hence $p_{2} \circ \ldots p_{m}\left(a^{\prime}\right)=0$ and $a=p^{m-1} a^{\prime}=i_{m} \circ \ldots i_{2} \circ p_{2} \circ \ldots p_{m}\left(a^{\prime}\right)=0$.

Assume that all $(,)_{r}$, for $r<m$, are perfect. Let $\chi \in \operatorname{Hom}_{\text {cont }}\left(B_{m}, \mathbb{Z} / p^{m} \mathbb{Z}\right)$. Then the continuous homomorphism $p_{m} \circ \chi: B_{m} \mapsto \mathbb{Z} / p^{m-1} \mathbb{Z}$ vanishes on $p^{m-1} B_{m}$. Since $B_{m} / p^{m-1} B_{m}$ is homeomorphic to $B_{m-1}$ via $p_{m}$, we obtain a continuous homomorphism $\chi^{\prime}: B_{m-1} \rightarrow \mathbb{Z} / p^{m-1} \mathbb{Z}$. By the induction assumption there is $a \in A_{m-1}$ such that $p_{m}(\chi(b))=\chi^{\prime}\left(p_{m}(b)\right)=\left(a, p_{m}(b)\right)_{m-1}=p_{m}\left(\left(i_{m}(a), b\right)_{m}\right)$ for all $b \in B_{m}$. Hence $\chi=\zeta_{m}\left(i_{m}(a)\right)+p^{m-1} \chi^{\prime \prime}$ for a continuous homomorphism $\chi^{\prime \prime}: B_{m} / p B_{m} \rightarrow \mathbb{Z} / p \mathbb{Z}$. Since $B_{m} / p B_{m}$ is homeomorphic to $B_{1}$ via $p_{2} \circ \cdots \circ p_{m}$, we get the corresponding continuous homomorphism $\chi^{\prime \prime \prime}: B_{1} \rightarrow \mathbb{Z} / p \mathbb{Z}$. Hence it is $\zeta_{1}\left(a^{\prime}\right)$ for some $a^{\prime} \in A_{1}$. Then $\chi^{\prime \prime}(b)=\chi^{\prime \prime \prime}\left(p_{2} \circ \cdots \circ p_{m}(b)\right)=\left(a^{\prime}, p_{2} \circ \cdots \circ p_{m}(b)\right)_{1}=\left(i_{m} \circ \cdots \circ i_{2}\left(a^{\prime}\right), b\right)_{m}$, so $\chi^{\prime \prime}=\zeta_{m}\left(i_{m} \circ \cdots \circ i_{2}\left(a^{\prime}\right)\right)$ and $\chi$ is in the image of $\zeta_{m}$.

\subsection{Special Mackey functors.}

Definition 3.6. Let $G$ be a profinite group. Recall that $B(G)$ is the category of finite left $G$-sets with continuous action of $G$. Its objects are the finite left $G$-sets, so each of them is isomorphic to a finite disjoint union of the set of all cosets $G / H_{i}, H_{i}$ is an open subgroup of $G$. Its morphisms are $G$-equivariant maps. Each such morphism between connected objects, i.e. $j: G / K \rightarrow G / H$ for two open subgroups $K, H$ of $G$, is the composite of the canonical surjection $s_{K / h^{-1} H h}: G / K \rightarrow G / h^{-1} H h$, where $K$ is an open subgroup of $h^{-1} H h$ for some $h \in G$, and of $c_{h}: G / h^{-1} H h \rightarrow G / H, g h^{-1} H h \mapsto g h^{-1} H$.

Let $\mathscr{A}=\left(\mathscr{A}^{*}, \mathscr{A}_{*}\right)$ be a Mackey functor, also satisfying (M5) below, from the category $B(G)$ to the category of abelian groups endowed with translation invariant topology (not necessarily making objects topological groups) and continuous closed homomorphisms between them.

This means that

$\mathscr{A}_{*}$ is a covariant functor and $\mathscr{A}^{*}$ is a contravariant functor,

(M2) for every object $R$ in the first category its $\mathscr{A}^{*}$-image coincides with its $\mathscr{A}_{*}$-image, denote it $\mathscr{A}(R)$,

(M3) the image of the disjoint union of objects of $B(G)$ is the direct sum of the images,

(M4) for every left (pullback) cartesian diagram in $B(G)$ the right diagram is commutative
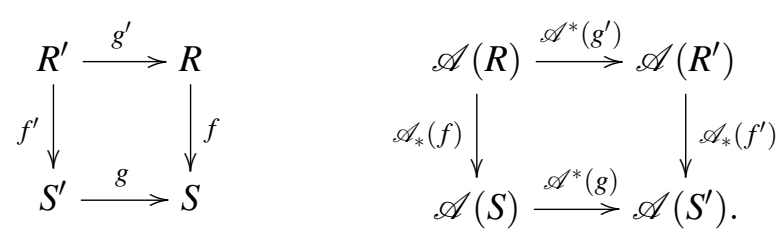

(M4) implies that if $K$ is a normal open subgroup of an open subgroup $H$ of $G$ and $f: G / K \rightarrow G / H$ is the canonical surjection, then $\mathscr{A}^{*}(f) \circ \mathscr{A}_{*}(f)=\sum \mathscr{A}^{*}\left(c_{h}\right)$ where $c_{h}: G / K \rightarrow G / K, h$ runs through representatives of $H / K$.

In addition, we will also assume 
(M5) if $K$ is an open subgroup of an open subgroup $H$ of $G$ and $f: G / K \rightarrow G / H$ is the canonical surjection, then

$$
\mathscr{A}_{*}(f) \circ \mathscr{A}^{*}(f)=|H: K| \operatorname{id}_{\mathscr{A}(G / H)}
$$

For an object $R=G / H$ of $B(G)$, with an open subgroup $H$ of $G$, we will use the notation

$$
\mathscr{A}(H):=\mathscr{A}(R) .
$$

Due to (M3), to describe a Mackey functor $\mathscr{A}$ on objects of $B(G)$ it suffices to indicate $H \mapsto \mathscr{A}(H), j \mapsto$ $\mathscr{A}_{*}(j), \mathscr{A}^{*}(j)$ satisfying (M1), (M2), (M5), and then extend to all objects and morphisms of $B(G)$ so that (M3), (M4) are satisfied.

For an open subgroup $H$ of $G$ denote by $H^{\text {ab }}$ its maximal abelian quotient.

Remark 3.7. Given a Mackey functor and replacing the topologies with the discrete topologies, we get a Mackey functor.

Example 3.8. One of first examples of Mackey functor is given by the functor which to $H$ associates the maximal abelian quotient $H^{\text {ab }}$, and to a $B(G)$-morphism $j=c_{h} \circ s_{K / h^{-1} H h}: G / K \rightarrow G / H$, as in Definition 3.6, associates $j_{*}: K^{\mathrm{ab}} \rightarrow H^{\mathrm{ab}}$ as the composite of the restriction and the conjugation by $h$ and $j^{*}: H^{\mathrm{ab}} \rightarrow K^{\mathrm{ab}}$ as the composite of the conjugation by $h^{-1}$ and the transfer (die Verlagerung) map between abelian groups. The $j_{*}, j^{*}$ are well defined.

Remark 3.9. For a Mackey functor $\mathscr{A}$ we have the Mackey functor $\mathscr{A}^{\odot}: H \mapsto \mathscr{A}(H)^{\odot}, j \mapsto \mathscr{A}^{*}(j)^{\odot}, \mathscr{A}_{*}(j)^{\odot}$.

Remark 3.10. If $A$ is a discrete topological $G$-module, then the functor $\mathscr{A}$ extending $H \mapsto A_{H}$ where $A_{H}$ is the $H$-fixed submodule of $A$, is a Mackey functor. For an open subgroup $K$ of an open subgroup $H$ of $G$, the canonical surjection $f: G / K \rightarrow G / H$ yields the norm morphism $N_{H / K}=\mathscr{A}_{*}(f): A_{K} \rightarrow A_{H}$ and the morphism $i_{H / K}=\mathscr{A}^{*}(f): A_{H} \rightarrow A_{K}$.

In particular, if $G=G(l / k)$ then $H \mapsto l_{H}$, where $l_{H}$ is the additive (resp. multiplicative) group of the fixed field of $H$, extends to a Mackey functor.

If $A$ is a $G$-module endowed with an appropriate topology such that the morphisms $i_{H / K}: A_{H} \rightarrow A_{K}$ and $N_{H / K}: A_{K} \rightarrow A_{H}$ are closed continuous, then again we get Mackey functors as just described.

General Mackey functors do not originate from $G$-modules $A$ as above. Various examples can be found in [38]. For example, if $A$ is as in the first paragraph, then $H \mapsto H^{i}(H, A)$, associated to the Galois cohomology $H^{i}$, is a Mackey functor. A relevant example for higher class field theory is $G$ being the Galois group of a Galois field extension $l / k$, then for any Milnor $K_{r}$-functor, the functor extending $H \mapsto K_{r}\left(l_{H}\right)$ is a Mackey functor, even though no Galois descent property holds for $K_{2}$ in general.

3.3. Mackey functors for fields of positive characteristic. Let $k$ be a field of characteristic $p>0$ as in the previous section, $G=G^{p}(k)=G\left(k^{\mathrm{sep}, p} / k\right)$.

For a closed subgroup $H$ of $G$ denote by $k_{H}$ the $H$-fixed subfield of $k^{\mathrm{sep}, p}$ and by $H^{\mathrm{ab}, p^{m}}$ the quotient of $H^{\mathrm{ab}}$ by the closure of its subgroup $\left(H^{\mathrm{ab}}\right)^{p^{m}}$, so $H^{\mathrm{ab}, p^{m}}$ is the maximal abelian topological quotient of $H$ such that all of its elements are of exponent $\leqslant p^{m}$.

Remark 3.11. For open subgroups $K, H$ of $G$ let $j=c_{h} \circ s_{K / h^{-1} H h}: G / K \rightarrow G / H$ be a $B(G)$-morphism as in Definition 3.6. Define $W_{*}(j)$ as the composite of the trace map and action by $h$ on Witt vectors and $W^{*}(j)$ as the composite of action by $h^{-1}$ and induced by the field embedding. Define similarly $\mathscr{W}_{*}(j), \mathscr{W}^{*}(j)$. Extend 
to direct sums of objects. If we endow $W_{m}$ and $\mathscr{W}$ with the discrete topology, then we have Mackey functors extending $H \mapsto W_{m}\left(l_{H}\right)$ and $H \mapsto \mathscr{W}\left(l_{H}\right)$.

Due to Witt theory, the functor of Example 3.8 is compatible with the functor $\mathscr{W}^{\circ}$ : the isomorphisms $\mathfrak{w}_{k_{H}}^{-1}: \mathscr{W}\left(k_{H}\right)^{\circ} \longrightarrow H^{\mathrm{ab}}$, see Proposition 2.6, satisfy the natural functorial properties expressed by the following commutative diagrams
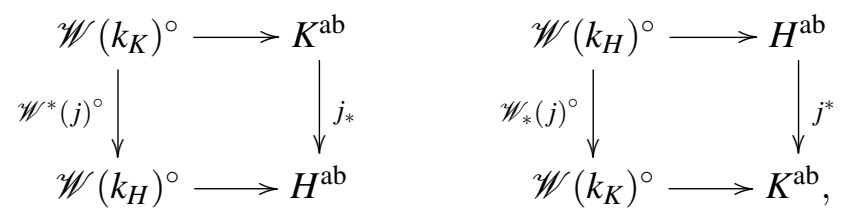

where for a morphism $g$ in the category of locally compact abelian groups the morphism $g^{\circ}$ is its dual.

Definition 3.12. (Topological assumptions on Witt objects and morphisms)

Assume that for all open subgroups $H$ of $G$ the additive group of the field $k_{H}$ is endowed with a translation invariant Hausdorff topology (not necessarily making the additive group $k_{H}$ a topological group). Consider on $W\left(k_{H}\right)$ and $W_{m}\left(k_{H}\right)$ the induced topologies from the inverse limit topology on the product of countably many copies of $k_{H}$. Assume that endomorphisms of $W\left(k_{H}\right)$ in the $k_{H}$-algebra generated by the identity map, $\mathbf{V}, \mathbf{F}$ are closed continuous. In particular, $\wp\left(W_{m}\left(k_{H}\right)\right)$ is closed in $W_{m}\left(k_{H}\right)$. Consider the induced topology

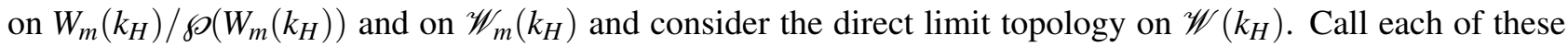
topologies on $W\left(k_{H}\right), W_{m}\left(k_{H}\right), W_{m}\left(k_{H}\right) / \wp\left(W_{m}\left(k_{H}\right)\right), \mathscr{W}_{m}\left(k_{H}\right)$ 'current topologies'. Extend to direct sums of objects by using direct product topologies.

Assume that for every morphism $j: G / K \rightarrow G / H$, the morphisms $W_{*}(j), W^{*}(j), \mathscr{W}_{*}(j), \mathscr{W}^{*}(j)$ defined in Remark 3.11 are continuous and closed with respect to the current topologies. Then we have new Mackey functors, with respect to the current topology, extending $H \mapsto W_{m}\left(l_{H}\right)$ and $H \mapsto \mathscr{W}\left(l_{H}\right)$.

Assume that the Witt pairings of Propositions 2.1, 2.6 are continuous in each variable with the additive Witt groups endowed with the current topologies.

We have $p_{m}: W_{m}(k) / \wp\left(W_{m}(k)\right) \rightarrow W_{m-1}(k) / \wp\left(W_{m-1}(k)\right)$ and $i_{m}$ in the opposite direction, see (e) of Examples 3.2. Due to the definition of the current topologies, they are continuous closed.

Due to the last sentence of Definition 3.12, we get a homomorphism $G^{a b, p}(k) \longrightarrow \mathscr{W}(k)^{\odot}$.

The following properties are immediate.

Lemma 3.13. The natural homomorphism $V^{\odot} \rightarrow V^{\circ}$ is continuous injective.

Assume all the properties of Definition 3.12. The isomorphism $\mathfrak{w}_{k}: G^{a b, p}(k) \longrightarrow \mathscr{W}(k)^{\circ}$ of Proposition 2.6 factorises into the composite of $G^{a b, p}(k) \longrightarrow \mathscr{W}(k)^{\odot}$ and the injective homomorphism $\mathscr{W}(k)^{\odot} \rightarrow \mathscr{W}(k)^{\circ}$. Hence $G^{a b, p}(k) \longrightarrow \mathscr{W}(k)^{\odot}$ is an algebraic isomorphism and so is $\mathscr{W}(k)^{\odot} \rightarrow \mathscr{W}(k)^{\circ}$. Similarly, the homomorphism $G\left({ }_{m} k / k\right) \longrightarrow\left(W_{m}(k) / \wp\left(W_{m}(k)\right)^{\odot}\right.$ is an algebraic isomorphism and so is $\left(W_{m}(k) / \wp\left(W_{m}(k)\right)^{\odot} \rightarrow\right.$ $\left(W_{m}(k) / \wp\left(W_{m}(k)\right)^{\circ}\right.$.

Definition 3.14. Fix a Mackey functor $\mathscr{A}$ from the category $B(G)$, satisfying (M1)-(M5). Assume that the properties of Definition 3.12 hold.

Then, in particular, due to (M5), the morphism $\mathscr{A}(H) \rightarrow \mathscr{A}(H)$ of multiplication by $p$ is continuous closed. Define on $\mathscr{A}(H) / p^{m} \mathscr{A}(H)$ the induced topology from $\mathscr{A}(H)$. The homomorphism $p_{m}: \mathscr{A}(H) / p^{m} \mathscr{A}(H) \rightarrow$ $\mathscr{A}(H) / p^{m-1} \mathscr{A}(H)$ is surjective continuous closed. Denote by $i_{m}: \mathscr{A}(H) / p^{m-1} \mathscr{A}(H) \rightarrow \mathscr{A}(H) / p^{m} \mathscr{A}(H)$ the homomorphism induced by multiplication by $p$, it is continuous closed. 
Suppose that the following four conditions hold:

(A1) For every open subgroup $H$ of $G$ and each $m \geqslant 1$ there are $\mathbb{Z} / p^{m} \mathbb{Z}$-bilinear pairings continuous in each variable

$$
(,)_{H, m}: \mathscr{A}(H) / p^{m} \mathscr{A}(H) \times W_{m}\left(k_{H}\right) \longrightarrow W_{m}\left(\mathbb{F}_{p}\right) \simeq \mathbb{Z} / p^{m} \mathbb{Z}
$$

such that $(a, \mathbf{F} w)_{H, m}=\mathbf{F}(a, w)_{H, m},(a, \mathbf{V} w)_{H, m}=\mathbf{V}(a, w)_{H, m-1}$ for all $a, w$.

Hence the pairing $(,)_{H, m}$ induces a continuous in each variable pairing (the additive Witt groups of $k_{H}$ are endowed with the current topology)

$$
\langle,\rangle_{H, m}: \mathscr{A}(H) / p^{m} \mathscr{A}(H) \times W_{m}\left(k_{H}\right) / \wp\left(W_{m}\left(k_{H}\right)\right) \longrightarrow W_{m}\left(\mathbb{F}_{p}\right) / \wp\left(W_{m}\left(\mathbb{F}_{p}\right)\right)=W_{m}\left(\mathbb{F}_{p}\right) \simeq \mathbb{Z} / p^{m} \mathbb{Z} .
$$

(A2) The sequences $\mathscr{A}(H) / p^{m} \mathscr{A}(H)$ and $W_{m}\left(k_{H}\right) / \wp\left(W_{m}\left(k_{H}\right)\right)$ are pairable with respect to $\langle,\rangle_{H, m}$.

(A3) $\left\langle\mathscr{A}_{*}(j)(a), w\right\rangle_{H, m}=\left\langle a, W^{*}(j)(w)\right\rangle_{K, m}, \quad\left\langle\mathscr{A}^{*}(j)(a), w\right\rangle_{K, m}=\left\langle a, W_{*}(j)(w)\right\rangle_{H, m}$ for all $a, w$ and morphisms $j$.

(A4) For $m=1$ the pairing $\langle,\rangle_{H, m}$ is algebraically perfect in each variable, i.e. each of the groups is isomorphic to the group of continuous homomorphisms from the other group to $\mathbb{Z} / p^{m} \mathbb{Z}$.

We will now study implications for $\mathscr{A}$ satisfying Definition 3.14.

Using Lemma 3.5, we see that (A1)-(A4) imply

(A5) for all $m \geqslant 1$ the pairing $\langle,\rangle_{H, m}$ is algebraically perfect.

Corollary 3.15. Assuming that the properties of Definition 3.14 hold, we get algebraic isomorphisms

$$
\mathscr{A}(H) / p^{m} \mathscr{A}(H) \simeq\left(W_{m}\left(k_{H}\right) / \wp\left(W_{m}\left(k_{H}\right)\right)\right)^{\odot}, \quad W_{m}\left(k_{H}\right) / \wp\left(W_{m}\left(k_{H}\right)\right) \simeq\left(\mathscr{A}(H) / p^{m} \mathscr{A}(H)\right)^{\odot} .
$$

Combining with the algebraic isomorphism $\left(W_{m}(k) / \wp\left(W_{m}(k)\right)^{\odot} \simeq\left(W_{m}(k) / \wp\left(W_{m}(k)\right)^{\circ}\right.\right.$ of Lemma 3.13, we get the algebraic isomorphism

$$
\mathscr{A}(H) / p^{m} \mathscr{A}(H) \simeq\left(W_{m}\left(k_{H}\right) / \wp\left(W_{m}\left(k_{H}\right)\right)\right)^{\circ} .
$$

Using Witt theory, we get the algebraic isomorphism

$$
\Psi_{H, m}: \mathscr{A}(H) / p^{m} \mathscr{A}(H) \simeq H^{\mathrm{ab}, p^{m}} .
$$

Remark 3.16. The continuity of $\mathscr{A}(H) / p^{m} \mathscr{A}(H) \longrightarrow\left(W_{m}\left(k_{H}\right) / \wp\left(W_{m}\left(k_{H}\right)\right)^{\odot}\right.$ will follow from Theorem 3.19 below. The Galois group is compact, so if $\mathscr{A}(H) / p^{m} \mathscr{A}(H)$ is not compact, then the isomorphism $\Psi_{H, m}$ is not a homeomorphism. For instance, it is not a homeomorphism for $n$-dimensional local fields, $n>1$, see Remark 4.16.

Remark 3.17. Conditions (A1)-(A4) of Definition 3.14 can be viewed as generalised Kawada-Satake class formation conditions for Mackey functors in class field theory in positive characteristic. One can easily state similar conditions for another theory available instead of Witt theory. For example, if $k$ contains an algebraically closed field of characteristic zero then one can use Kummer theory.

Put

$$
\mathscr{C}(H)=\mathscr{A}(H) /\left(\cap_{m \geqslant 1} p^{m} \mathscr{A}(H)\right)
$$

with the induced topology from $\mathscr{A}(H)$. Morphisms $\mathscr{A}^{*}(j), \mathscr{A}_{*}(j)$ induce morphisms $\mathscr{C}^{*}(j), \mathscr{C}_{*}(j)$. 


\subsection{Class field theory mechanism.}

Theorem 3.18. Assume that the properties of Definition 3.14 hold. Then for every open subgroup $H$ of $G$ we get a continuous and non-degenerate in each variable pairing

$$
\langle,\rangle_{H}: \mathscr{C}(H) \times \mathscr{W}\left(k_{H}\right) \longrightarrow \mathbb{Q}_{p} / \mathbb{Z}_{p}
$$

It induces an injective homomorphism, the ( $p$-primary) $H$-reciprocity homomorphism

$$
\Phi_{H}: \mathscr{C}(H) \longrightarrow H^{\mathrm{ab}} .
$$

The functor $\mathscr{C}$ is compatible with the functor of Example 3.8: for a $B(G)$-morphism $j: G / K \rightarrow G / H$ with open subgroups $K, H$ of $G$, the diagrams
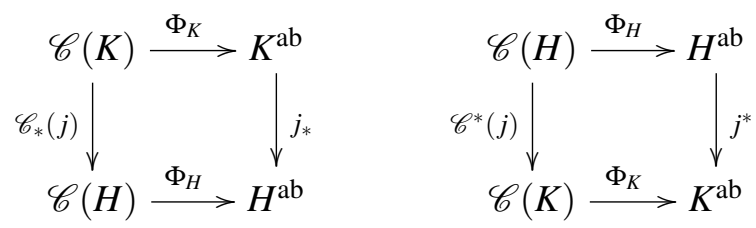

are commutative.

Proof. Due to (A1)-(A5) for every open subgroup $H$ of $G$ we get the continuous and non-degenerate in each variable pairing $\langle,\rangle_{H}$ and hence an injective homomorphism $\mathscr{C}(H) \longrightarrow \mathscr{W}\left(k_{H}\right)^{\odot}$. Its composition with the continuous algebraic isomorphism $\mathscr{W}\left(k_{H}\right)^{\odot} \longrightarrow \mathscr{W}\left(k_{H}\right)^{\circ}$, see Lemma 3.13, and the isomorphism $\mathfrak{w}_{k}^{-1}: \mathscr{W}(k)^{\circ} \longrightarrow G^{a b, p}(k)$ of Proposition 2.6, yields an injective homomorphism, the ( $p$-primary) $H$-reciprocity homomorphism

$$
\Phi_{H}: \mathscr{C}(H) \longrightarrow H^{\mathrm{ab}} .
$$

Property (A3) implies commutative diagrams
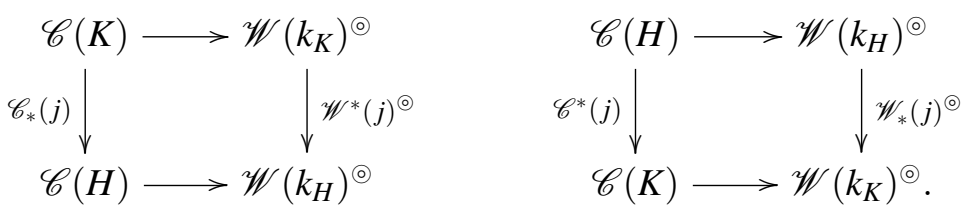

Remark 3.11 and Lemma 3.13 imply the rest.

For a normal open subgroup $K$ of $H$ and the canonical surjection $j: G / H \rightarrow G / K$, denote $N_{H / K}:=\mathscr{A}_{*}(j)$.

Condition (A3) of Definition 3.14 implies that the image $\Phi_{H}\left(N_{H / K} \mathscr{C}(K)\right)$ is in $K$.

Hence the reciprocity homomorphism $\Phi_{H}$ induces the homomorphism

$$
\Phi_{H / K}: \mathscr{C}(H) / N_{H / K} \mathscr{C}(K) \longrightarrow(H / K)^{\mathrm{ab}} .
$$

Theorem 3.19. Assume that the properties of Definition 3.14 hold.

Let $K$ be a normal open subgroup of $H$. Then the index of norm group $N_{H / K} \mathscr{C}(K)$ in $\mathscr{C}(H)$ equals the order of $(H / K)^{\mathrm{ab}}$ and the reciprocity homomorphism $\Phi_{H}$ induces the isomorphism

$$
\Phi_{H / K}: \mathscr{C}(H) / N_{H / K} \mathscr{C}(K) \simeq(H / K)^{\mathrm{ab}}
$$

Assume that $H / K$ is abelian of exponent $p^{i}, i \leqslant m$. Let $k_{K}=k_{H}\left(\wp^{-1}(V)\right)$, where $V$ is a closed subgroup of $W_{m}\left(k_{H}\right)$ containing $\wp\left(W_{m}\left(k_{H}\right)\right)$. Then the norm group $N_{H / K} \mathscr{C}(K)$ is the complement of $V$ with respect to the pairing $\langle,\rangle_{H, m}$ of Definition 3.14.

The homomorphism $\Phi_{H}$ is continuous and its image is everywhere dense in $H^{\mathrm{ab}}$. 
Proof. The homomorphisms $\Psi_{H, m}: \mathscr{A}(H) / p^{m} \mathscr{A}(H) \simeq H^{\mathrm{ab}, p^{m}}$ of Corollary 3.15 are compatible with the functor of $\mathscr{A} / p^{m} \mathscr{A}$ and the functor of Example 3.8. In particular, the following diagram is commutative

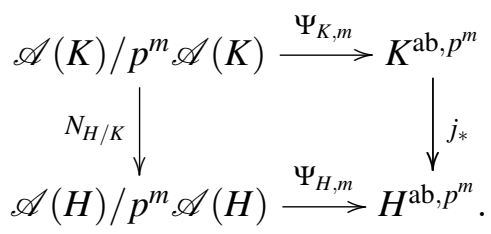

Hence $\mathscr{A}(H) / N_{H / K} \mathscr{A}(K) \simeq(H / K)^{\mathrm{ab}}$.

Denote by $V^{\perp}$ the complement of $V$. Property (A5) in subsection 3.3 implies that we have the non-degenerate pairing of finite abelian group $\mathscr{A}(H) / V^{\perp} \times V / \wp\left(W_{m}\left(k_{H}\right)\right) \rightarrow \mathbb{Z} / p^{m} \mathbb{Z}$, hence they have the same order, so $\left|\mathscr{A}(H): V^{\perp}\right|=|H: K|$. On the other hand, $N_{H / K} \mathscr{A}(K)$ is a subgroup of $V^{\perp}$. Since $N_{H / K} \mathscr{C}(K)$ and $V^{\perp}$ have equal indices, we deduce $N_{H / K} \mathscr{C}(K)=V^{\perp}$. Thus, the pairing $\langle,\rangle_{H, m}$ induces the non-degenerate pairing $\mathscr{A}(H) / V^{\perp} \times V / \wp\left(W_{m}\left(k_{H}\right)\right) \rightarrow \mathbb{Z} / p^{m} \mathbb{Z}$ and $\Phi_{H / K}$ is an isomorphism.

The equality $\Phi_{H}^{-1}(K)=N_{H / K} \mathscr{A}(K)$ implies the last sentence of the theorem.

Theorem 3.20. (Existence theorem) Assume that the properties of Definition 3.14 hold. The lattice of open subgroups $K$ of $H^{\mathrm{ab}}$ (with respect to the intersection and the product) is in an order preserving bijection with the lattice of closed subgroups of finite index in $\mathscr{C}(H)$ (with respect to the intersection and the product): $K \mapsto$ $N_{H / K} \mathscr{A}(K)$.

Proof. Norm subgroups of finite abelian extensions are closed of finite index in view of the second paragraph of Theorem 3.19. We will show that closed subgroups of finite index are norm subgroups.

Let $\mathscr{N}$ be a closed subgroups of finite index of $\mathscr{A}(K) / p^{m} \mathscr{A}(K)$, and let $V$ be its complement with respect to the pairing $\langle,\rangle_{H, m}$. By Theorem 3.19 we know that $V^{\perp}=N_{H / K} \mathscr{A}(K)$, where $K$ is an open subgroup of $H$ which corresponds to $V$ via Witt theory. Since $\mathscr{N}$ is a subgroup of $V^{\perp}, \mathscr{N} \leqslant N_{H / K} \mathscr{A}(K)$. On the other hand, for every $b \in N_{H / K} \mathscr{A}(K)$ the automorphism $\Phi_{H, m}(b)$ acts trivially on the field corresponding to $V$ via Witt theory. Hence $\langle b, V\rangle_{H, m}=0$, so $N_{H / K} \mathscr{A}(K)$ is a subgroup of the double complement $\mathscr{N}^{\perp \perp}$ of $\mathscr{N}$. Thus, $\mathscr{N} \leqslant N_{H / K} \mathscr{A}(K) \leqslant \mathscr{N}^{\perp \perp}$.

In particular, if $\mathscr{N}$ is a closed subgroups of index $p$ in $\mathscr{A}(K) / p^{m} \mathscr{A}(K)$ then either have $\mathscr{N}=\mathscr{N}^{\perp \perp}$ and then by the previous material $\mathscr{N}=N_{H / K} \mathscr{A}(K)$, or we have $\mathscr{N}^{\perp \perp}=\mathscr{A}(H)$, which is impossible, since property (A4) then implies $\mathscr{N}^{\perp}=0$. Thus, every closed subgroup of $\mathscr{A}(H)$ of prime index is the norm group.

Now use the standard induction on $m \geqslant 1$ to show that any closed subgroup $\mathscr{N}$ of index $p^{m}$ contains a norm subgroup. Let $m>1$, and let $\mathscr{N}^{\prime}$ be an closed subgroup of index $p^{m-1}$ such that $\mathscr{N}<\mathscr{N}^{\prime}$. By the induction assumption, $\mathscr{N}^{\prime} \geqslant N_{H / K^{\prime}} \mathscr{A}\left(K^{\prime}\right)$ for a normal open subgroup $K^{\prime}$ of $H$. The subgroup $\mathscr{N} \cap N_{H / K^{\prime}} \mathscr{A}\left(K^{\prime}\right)$ is either of index 1 or $p$ in $N_{H / K^{\prime}} \mathscr{A}\left(K^{\prime}\right)$. In the first case $\mathscr{N} \geqslant N_{H / K^{\prime}} \mathscr{A}\left(K^{\prime}\right)$, and in the second case let $K$ be an open subgroup of $K^{\prime}$ such that $N_{H / K^{\prime}}^{-1}\left(\mathscr{N} \cap N_{H / K^{\prime}} \mathscr{A}\left(K^{\prime}\right)\right) \geqslant N_{K^{\prime} / K} \mathscr{A}(K)$, then $\mathscr{N} \geqslant N_{H / K} \mathscr{A}(K)$.

Now, since $\mathscr{N} \geqslant N_{H / K} \mathscr{A}(H)$, by Theorem 3.19 the group $\mathscr{N}$ includes $N_{H / L} \mathscr{A}(L)$ where $k_{L}$ is the maximal abelian extension of $k_{H}$ in $k_{K}$. Then by Theorem $3.19 \mathscr{N}=N_{H / M} \mathscr{A}(M)$ where $M$ is is the fixed field of $\Phi_{H / L}(\mathscr{N})$, as required.

The rest follows by the usual arguments in class field theory, see e.g. [31, Ch.II sect.4].

\subsection{Remarks and Examples.}

Remark 3.21. Observe that all the proofs in the preceding part (class field theory mechanism) of this section are group theoretical. The use of fixed fields $k_{H}$ of open subgroups $H$ of $G$ was just for convenience purposes. 
Remark 3.22. Topological duality and existence theorem. Theorems 3.19 and 3.20 imply that every closed subgroup $\mathscr{N}$ of finite index $p^{r}$ of $\mathscr{A}(H)$ such that the quotient group is of exponent $p^{i}, i \leqslant m$, and every finite subgroup $V$ of $W_{m}\left(k_{H}\right) / \wp\left(W_{m}\left(k_{H}\right)\right)$ are reflexive with respect to the pairing $\langle,\rangle_{H, m}$ of Definition 3.14: $\mathscr{N}=\mathscr{N} \perp \perp$ and $V=V^{\perp \perp}$, and there is duality between finite discrete abelian groups $\mathscr{A}(H) / \mathscr{N} \times \mathscr{N}^{\perp} / \wp\left(W_{m}\left(k_{H}\right)\right) \rightarrow$ $\mathbb{Z} / p^{m} \mathbb{Z}$. On the other hand, due to the second paragraph of the proof of Theorem 3.20, every reflexive (with respect to $\langle,\rangle_{H}$ ) closed subgroup of finite index of $\mathscr{C}(H)$ is the norm group. Thus, reflexivity of closed subgroups of $\mathscr{C}(H)$ of finite index is closely related to the existence theorem, a key component of class field theory. This gives a topological duality perspective on the meaning of the existence theorem.

Remark 3.23. The properties of Definition 3.14 are quite different from standard class formation axioms used in the previous methods to study class field theory mechanisms, starting with [1] and its modifications, including the Neukirch method [31], [32] and the generalised methods of [4], [6]. The main difference is that no requirements on the index of the norm group or the kernel of the norm map and no the requirement for the reciprocity homomorphism to be an isomorphism for extensions of prime degree is explicitly included in these axiomatic properties for the generalised Kawada-Satake approach in this paper.

Remark 3.24. Since non-degenerate pairings are important for this approach to class field theory, we include an observation which helps to establish their non-degeneracy.

Let $B$ be a commutative Hausdorff topological ring. Let $X$ be an $B$-module. Suppose that $X$ is endowed with a topology which is translation invariant. Suppose that the structure map $B \times X \rightarrow X$ is continuous. Then we call $X$ a topological $B$-module. Let $I$ be a totally ordered countable set. A set $\left\{x_{i}\right\}_{i \in I}$ of elements of $X$ is called a set of topological generators of the $B$-module $X$ if every element $x \in X$ can be expressed as a convergent series $\sum_{i \leqslant j} a_{i} x_{i}$ with some $a_{i} \in B$, where the order of terms of the series corresponds to - the ordering of $I$. A set of topological generators $\left\{x_{i}\right\}_{i \in I}$ of $X$ is called a B-topological basis of $X$ if for every $j \in I$ the condition that a convergent series $\sum_{i \leqslant j} a_{i} x_{i}$, with $a_{i} \in B$, is zero implies all $a_{i}=0$. If $\left\{x_{i}\right\}_{i \in I}$ is a topological basis of $X$ then every element $x \in X$ can be uniquely expressed as a convergent series $\sum_{i \leqslant j} a_{i} x_{i}$ with some $a_{i} \in B, j \in J$.

Let $X, Y$ be topological $B$-modules. Assume that there is a continuous $B$-bilinear pairing

$$
\langle,\rangle: X \times Y \longrightarrow B
$$

Assume that $Y$ has topological generators $\left\{y_{i}\right\}_{i \in I}$. Assume that for every $j \in I$ there is an element $x_{j} \in X$ such that

$$
\left\langle x_{j}, y_{j}\right\rangle=1, \quad\left\langle x_{j}, y_{i}\right\rangle=0 \quad \text { for all } i>j .
$$

Suppose that a convergent sum $\sum_{i \leqslant j} c_{i} y_{i}$ equals 0 . Then $0=\sum_{i} c_{i}\left\langle x_{j}, y_{i}\right\rangle=c_{j}$. Thus, $\left\{y_{i}\right\}_{i \in I}$ form a topological basis of $Y$ and the pairing $\langle$,$\rangle is non-degenerate with respect to y \in Y$.

\subsection{Class field theories of one-dimensional fields of positive characteristic.}

Example 3.25. One-dimensional local class field theory in positive characteristic. Let $F$ be a complete discrete valuation field of positive characteristic $p>0$ with a finite residue field. Its additive group has its standard topology. The group $F^{\times}$is endowed with the induced topology from the product topology on $F \times F$, via $\alpha \mapsto\left(\alpha, \alpha^{-1}\right)$.

The Mackey functor $\mathscr{A}$ associates to an open subgroup $H$ of $G^{\text {sep, } p}(F)$ the multiplicative group $F_{H}^{\times}$of the local field $F_{H}$ that is the $H$-fixed subfield of $F^{\text {sep }}$. Instead of using groups, we will use their fixed fields. For a field embedding $i_{L / M}: L \rightarrow M$, the morphism $N_{M / L}=\mathscr{A}_{*}\left(i_{L / M}\right): M^{\times} \rightarrow L^{\times}$is induced by the norm map and $\mathscr{A}_{*}\left(i_{L / M}\right): L^{\times} \rightarrow M^{\times}$is induced by $i_{L / M}$. 
The current topology of $W(F)$, as in Definition 3.12, is defined as induced from the product topology of countably many copies of the additive topological group and topological $\mathbb{F}_{p}$-vector space $F$ with respect to $W(F)<\prod F$. So the topology of $W_{m}(F)$ is locally compact. Since $M_{F}$ is a subset of $\wp(F)$, it is straightforward that the current topology of $W_{m}(F) / \wp\left(W_{m}(F)\right)$ is the discrete topology.

Properties of Definition 3.12 hold true.

Choose a prime element $\pi$ of the field $F=F_{H}$, with the ring of integers $O_{F}$, its maximal ideal $M_{F}$ and the residue field $\mathbb{F}_{q}$. The group $F^{\times}$is isomorphic to $\mathbb{Z} \oplus \mathbb{F}_{q}^{\times} \oplus U_{1, F}$ where $\mathbb{Z}$ corresponds to the infinite cyclic group generated by $\pi, U_{1, F}=1+M_{F}$.

The group of principal units $U_{1, F}$ is a topological $\mathbb{Z}_{p}$-module $\left(\mathbb{Z}_{p}\right.$ is equipped with its standard $p$-adic topology) with a countable set of topological generators $1+\theta_{i} \pi^{s}$, where $s \geqslant 1,(s, p)=1$, and $\theta_{i} \in O_{F}^{\times}$, $1 \leqslant i \leqslant f=\log _{p} q$, are such that their images in the residue field form a basis over $\mathbb{F}_{p}$ and $\theta_{1}=1$, see e.g. [15, Ch.I sect.6]. Choose elements $\eta_{j} \in O_{F}^{\times}, 1 \leqslant j \leqslant f$, whose images in $\mathbb{F}_{q}$ form the dual basis of $\mathbb{F}_{q}$ over $\mathbb{F}_{p}$ with respect to the previous basis. It is easy to see that the group $F / \wp(F)$ is a discrete group with topological generators $\eta_{1}, \eta_{j} \pi^{r}$, where $1 \leqslant j \leqslant f$ and $r<0,(r, p)=1$.

Denote $O_{0}=W\left(\mathbb{F}_{q}\right)$, then we have a surjective homomorphism $p: O_{0} \rightarrow \mathbb{F}_{q},\left(w_{0}, \ldots\right) \mapsto w_{0}$. Recall that $F$ is isomorphic to the field of formal power series $\mathbb{F}_{q}((\pi))$. We get a surjective homomorphism (depending on the choice of $\pi$ )

$$
p_{\pi}: O_{0}((X)) \longrightarrow F, \quad \sum a_{i} X^{i} \mapsto \sum p\left(a_{i}\right) \pi^{i}
$$

This gives surjective maps $p_{\pi}: W_{m}\left(O_{0}((X))\right) \rightarrow W_{m}\left(\mathbb{F}_{q}((X))\right), p_{\pi}: O_{0}((X))^{\times} \rightarrow \mathbb{F}_{q}((X))^{\times}, \underline{\alpha} \mapsto \alpha$. To compute ghost components of elements of $W(F)$ we can use the corresponding lifts to the ring $O_{0}((X))$ of characteristic 0 .

For $m \geqslant 1$ the pairing $(,)_{H, m}$ is the Artin-Schreier-Witt pairing

$$
(,)_{m, H}: F^{\times} / F^{\times p^{m}} \times W_{m}(F) \longrightarrow W_{m}\left(\mathbb{F}_{p}\right) \simeq \mathbb{Z} / p^{m} \mathbb{Z}, \quad(\alpha, \beta)_{m, H}=\operatorname{Tr}_{\mathbb{F}_{q} / \mathbb{F}_{p}}(\gamma),
$$

where the $i$ th ghost component $\gamma^{(i)} \in O_{0}((X))$ of $\gamma$ is given by $\operatorname{res}\left(\beta^{(i)} \underline{\alpha}^{-1} d \underline{\alpha}\right)$ where $\beta^{(i)} \in O_{0}((X))$ is the $i$ th ghost component of $\underline{\beta}$. The value of the pairings does not depend on the lifts and the choice of $\pi$, the pairing is well defined. The properties (A1), (A2), (A3) of Definition 3.14 are satisfied.

Thus we get the induced pairing, also called the Artin-Schreier-Witt pairing

$$
\langle,\rangle_{m, H}: F^{\times} / F^{\times p^{m}} \times W_{m}(F) / \wp\left(W_{m}(F)\right) \longrightarrow W_{m}\left(\mathbb{F}_{p}\right) \simeq \mathbb{Z} / p^{m} \mathbb{Z} .
$$

It is continuous in each variable.

Elementary computations of the Artin-Schreier pairing show

$$
\begin{aligned}
& \left\langle\eta_{1}, \pi\right\rangle_{1}=1, \quad\left\langle\eta_{1}, 1+\theta_{i} \pi^{s}\right\rangle_{1}=0 \text { for } s>0, \quad\left\langle\eta_{j} \pi^{r}, \pi\right\rangle_{1}=0 \text { for } r<0, \\
& \left\langle\eta_{j} \pi^{r}, 1+\theta_{i} \pi^{s}\right\rangle_{1}=0 \text { if } s+r>0 \text { or } i \neq j, \quad\left\langle\eta_{j} \pi^{r}, 1+\theta_{i} \pi^{s}\right\rangle_{1}=1 \text { if } s=r, i=j .
\end{aligned}
$$

For the use of the Artin-Hasse exponential map for additional computations of $\langle,\rangle_{m}$ that are not needed in this paper see [35, sect.3.0].

These equalities and Remark 3.24 help to show that the two sets of topological generators are topological bases, and to deduce that the pairing $\langle,\rangle_{1}$ is non-degenerate. It is not difficult to prove the non-degeneracy of $\langle,\rangle_{m}$ directly, using properties of Witt vectors, and to deduce that $\left(\eta_{1}, 0, \ldots\right),\left(\eta_{j} \pi^{r}, 0, \ldots\right)$ form a topological basis of $W_{m}(F) / \wp\left(W_{m}(F)\right)$. Alternatively, property (A5) in subsection 3.3 shows that the pairing $\langle,\rangle_{m}$ is non-degenerate. 
It is easy to show that $\langle,\rangle_{m}$ is perfect, and yields a duality between the compact group $F^{\times} / F^{\times} p^{m}$ and the discrete group $W_{m}(F) / \wp\left(W_{m}(F)\right)$. The compact group $F^{\times} / F^{\times p^{m}}$ is isomorphic, as a topological group, to the group $\left(W_{m}(F) / \wp\left(W_{m}(F)\right)\right)^{\circ}$. Every closed subgroups of $F^{\times} / F^{\times} p^{m}$ of finite index is reflexive with respect to $\langle,\rangle_{m}$.

Thus, all the conditions of Definition 3.14 are satisfied. Via the class field theory mechanism in this section we obtain all the main theorems of local class field theory: Theorem 3.19 and Theorem 3.20. Thus, we get the most difficult and central part, the $p$-primary part of the local reciprocity homomorphism

$$
F^{\times} \longrightarrow G^{\mathrm{ab}, p}(F) .
$$

Its kernel is $\cap_{m \geqslant 0} F^{\times p^{m}}=\mathbb{F}_{q}^{\times}$.

Note that no Galois cohomology computations are needed or used in this presentation of local class field theory in positive characteristic. This presentation simplifies and straightens the arguments in [27] and [35].

To get the full local class field theory for $F$, one uses two other parts of the reciprocity homomorphism and then easily checks that they all glue together well. The other two parts are the unramified part with the homomorphism

$$
F^{\times} \longrightarrow G\left(F^{\mathrm{ur}} / F\right), \quad \alpha \mapsto v(\alpha) \in \mathbb{Z}<\hat{\mathbb{Z}},
$$

where $v$ is the surjective discrete valuation and $F^{\mathrm{ur}}$ is the maximal unramified extension of $F$, and the prime-to- $p$ Kummer class field theory

$$
F^{\times} \rightarrow G\left(F\left(\sqrt[q-1]{F^{\times}}\right) / F\right)
$$

which is derived in a similar way, but much easier since one works with the $(q-1)$-part, using Kummer theory and the tame symbol instead of Witt theory and the Artin-Schreier-Witt pairing. For the use of Kummer theory in explicit local class field theory see also [15, Ch.VIII sect.3] .

Example 3.26. One-dimensional global class field theory in positive characteristic. Let $K$ be an algebraic function field in one variable over a finite field $\mathbb{F}_{q}$ of characteristic $p>0$, i.e. $K$ is the function field of an irreducible curve over $\mathbb{F}_{q}$. Assume that $\mathbb{F}_{q}$ is algebraically closed in $K$.

The additive groups $K$ and $W_{m}(K)$ are endowed with the discrete topology, properties of Definition 3.12 hold true. The additive group of adeles $\mathbb{A}_{K}$ gets the induced topology from the product topology of the completions $K_{v}$ for all places $v$ of $K$, the group $W_{m}\left(\mathbb{A}_{K}\right)$ gets the induced topology from the product topology of $\mathbb{A}_{K}^{\oplus m}$. Algebraically and topologically, $W_{m}\left(\mathbb{A}_{K}\right)$ is the restricted product of $W_{m}\left(K_{v}\right)$ with respect to $W_{m}\left(O_{v}\right)$, where $O_{v}$ is the ring of integers of $K_{v}$, the subgroup $\wp\left(W_{m}\left(\mathbb{A}_{K}\right)\right)$ is the restricted product of $\wp\left(W_{m}\left(K_{v}\right)\right)$ with respect to $\wp\left(W_{m}\left(O_{v}\right)\right)$, while the quotient group $W_{m}\left(\mathbb{A}_{K}\right) / \wp\left(W_{m}\left(\mathbb{A}_{K}\right)\right)$ is the restricted product of $W_{m}\left(K_{v}\right) / \wp\left(W_{m}\left(K_{v}\right)\right)$ with respect to $\left(W_{m}\left(O_{v}\right)+\wp\left(W_{m}\left(K_{v}\right)\right)\right) / \wp\left(W_{m}\left(K_{v}\right)\right)$.

The Mackey functor $\mathscr{A}$ associates to an open subgroup $H$ of $G^{\text {sep, } p}(K)$ the idele class group $J_{K_{H}}=\mathbb{A}_{K_{H}}^{\times} / K_{H}^{\times}$ of the global field $K_{H}$ that is the $H$-fixed subfield of $K^{\text {sep. }}$. We have $J_{K} / J_{K}^{p^{m}}=\mathbb{A}_{K}^{\times} /\left(K^{\times} \mathbb{A}_{K}^{\times p^{m}}\right)$.

Instead of using groups, we will use their fixed fields. For a field embedding $i_{L / M}: L \rightarrow M$, the morphism $N_{M / L}=\mathscr{A}_{*}\left(i_{L / M}\right): J_{M} \rightarrow J_{L}$ is induced by the norm map and $\mathscr{A}_{*}\left(i_{L / M}\right): J_{L} \rightarrow J_{M}$ is induced by $i_{L / M}$.

Using the local Artin-Schreier-Witt pairings of Example 3.25 we get the adelic pairing

$$
\mathbb{A}_{K}^{\times} / \mathbb{A}_{K}^{\times p^{m}} \times W_{m}\left(\mathbb{A}_{K}\right) / \wp\left(W_{m}\left(\mathbb{A}_{K}\right)\right) \longrightarrow \mathbb{Z} / p^{m} \mathbb{Z}
$$

It is continuous and perfect in each variable. Condition (A1) of Definition 3.14 is satisfied. The classical reciprocity property for this pairing, [41], is that global elements go to 0. Looking at extensions which locally split everywhere, one immediately proves that $W_{m}(K) / \wp\left(W_{m}(K)\right)$ is isomorphic, as a topological group, to $\left(W_{m}(K)+W_{m}\left(\mathbb{A}_{K}\right)\right) / \wp\left(W_{m}\left(\mathbb{A}_{K}\right)\right)$. 
By restricting the previous pairing to the subgroup $\left(W_{m}(K)+W_{m}\left(\mathbb{A}_{K}\right)\right) / \wp\left(W_{m}\left(\mathbb{A}_{K}\right)\right)$ of the second group we get the continuous in each variable pairing

$$
\langle,\rangle_{m}: \mathbb{A}_{K}^{\times} /\left(K^{\times} \mathbb{A}_{K}^{\times p^{m}}\right) \times W_{m}(K) / \wp\left(W_{m}(K)\right) \longrightarrow \mathbb{Z} / p^{m} \mathbb{Z} .
$$

Conditions (A2)-(A3) of Definition 3.14 are satisfied for $\langle,\rangle_{m}$. It is not difficult to check that the pairing $\langle,\rangle_{1}$ is algebraically perfect, see e.g. [27, sect.3 (IV)]. Thus, all the conditions of Definition 3.14 are satisfied.

Theorem 3.18 now yields the $p$-primary part of the global reciprocity homomorphism

$$
\mathbb{A}_{K}^{\times} / K^{\times} \longrightarrow G^{\mathrm{ab}, p}(K) .
$$

Via the class field theory mechanism in this section we obtain the main theorems of the $p$-primary part of global class field theory of $K$ : Theorem 3.19 and Theorem 3.20.

Note that no Galois cohomology computations are needed or used in this presentation of local class field theory in positive characteristic. For other details see [27, sect.3] and [42].

To get the full local class field theory for $K$, one uses two other parts of the reciprocity homomorphism and then easily checks that they all glue together well. The other two parts are the unramified part with the constant field extension and the prime-to- $p$ part, i.e. the study of Kummer extensions of $K$ of exponent dividing $q-1$.

Remark 3.27. Further generalisations of the method. While the generalised Kawada-Satake class field theory mechanism of this section is applicable in positive characteristic to local fields with a finite residue field (Example 3.25) and function fields of curves over finite fields (Example 3.26), and to higher local fields whose last residue field is finite (section 4), it is not applicable to local fields with an arbitrary perfect residue field and to higher local fields with an arbitrary last perfect residue field.

One can modify the current method to make it work in more generality when the finite field is replaced with a perfect field of characteristic $p$ that is not separably $p$-closed. To achieve that, one can use the philosophy of [5] and [6]. Briefly writing, one needs to replace the pairing $(,)_{H, m}$ in Definition 3.14 by a pairing

$$
(,)_{H, m}: \mathscr{A}(H) / p^{m} \mathscr{A}(H) \times W_{m}\left(k_{H}\right) \longrightarrow \operatorname{Hom}_{\mathbb{Z}_{p}, \text { cont }}\left(G^{\mathrm{ur}, \mathrm{ab}, p}\left(k_{H}\right), W_{m}\left(\mathbb{F}_{p}\right)\right) \simeq\left(\mathbb{Z} / p^{m} \mathbb{Z}\right)^{\oplus \kappa},
$$

where $G^{\mathrm{ur}, \mathrm{ab}, p}\left(k_{H}\right)$ is the Galois group of the maximal unramified (maximal constant in the case of onedimensional global fields of characteristic $p$, maximal purely unramified in the case of higher local fields of characteristic $p$ ) $p$-primary abelian extension of $k_{H}, \kappa=\operatorname{dim}_{\mathbb{F}_{p}} k_{H} / \wp\left(k_{H}\right) \neq 0$, and $\operatorname{Hom}_{\mathbb{Z}_{p}, \text { cont }}$ stands for continuous $\mathbb{Z}_{p}$-homomorphisms. All the previous definitions and results in this section, except the existence theorem, generalise and yield the reciprocity homomorphism

$$
\Phi_{H}: \mathscr{C}(H) \longrightarrow \operatorname{Hom}_{\mathbb{Z}_{p}, \text { cont }}\left(G^{\mathrm{ur}, \mathrm{ab}, p}\left(k_{H}\right), H^{\mathrm{ab}}\right) .
$$

A generalisation of the existence theorem requires a more careful consideration of normic subgroups of $\mathscr{A}(H)$. It is not true in general that every closed subgroup of finite index is the norm group. For details see [5, sect.3].

\section{Class FIELD THEORY OF HIGHER LOCAL FIELDS OF POSITIVE CHARACTERISTIC}

\subsection{Higher local fields of positive characteristic.}

Definition 4.1. A one-dimensional local field is a complete discrete valuation field whose residue field is finite. An $n$-dimensional local field of positive characteristic $p$ is a complete discrete valuation field whose residue field is $(n-1)$-dimensional. Thus, to every $n$-dimensional local field of positive characteristic we can associate 
the sequence of its residue field, the residue field of the residue field, etc., ending with the so called 'last residue field' which is the finite field $\mathbb{F}_{q}$.

Such $n$-dimensional local fields of positive characteristic and their finite direct sums are associated, via iterated applications of operations of taking local rings, their completions and their fraction fields, to full flags of irreducible subschemes of an integral projective scheme over a finite field.

Definition 4.2. Let $\mathscr{O}_{F}$ be the ring of integers (of rank 1) of $F$ as a discrete valuation field. An ordered sequence $t_{n}, \ldots, t_{1}$ of elements of $\mathscr{O}_{F}$ is called local parameters of $F$ if $t_{n}$ is a prime element of $F$ as a discrete valuation field, $t_{i}$ for $i>1$ are units of $\mathscr{O}_{F}$ and their images in the residue field of $F$ are local parameters of the residue field $k_{n-1}$. Then $F$ is isomorphic to the formal power series field $\mathbb{F}_{q}\left(\left(t_{1}\right)\right) \ldots\left(\left(t_{n}\right)\right)$, due to applications of the structure theorem for complete discrete valuation fields, see e.g. [15, Ch.II sect.5].

Associated to $t_{n}, \ldots, t_{1}$ we have a discrete valuation $v_{n}$ of rank $n$ of $F$. It is a valuation homomorphism from $F^{\times}$to the lexicographically ordered (from the left) group $\mathbb{Z}^{\oplus n}$. Its value $\left(i_{n}, \ldots, i_{1}\right)$ on an element $\alpha \in F^{\times}$is computed as follows: $i_{n}$ is the discrete valuation (of rank 1) of $\alpha$, then consider the image of the element $\alpha t_{n}^{-i_{n}}$ in the residue field of $F$, its valuation of rank $n-1$ gives $\left(i_{n-1}, \ldots, i_{1}\right)$.

The ring of integers of $v_{n}$ is denoted by $O_{F}$, it does not depend on the choice of local parameters. Denote by $\mathscr{M}_{F}$ the maximal ideal of $\mathscr{O}_{F}$ and by $M_{F}$ the maximal ideal of $O_{F}$.

The group of principal units $V_{F}$ of $F$ is $1+M_{F}$. The choice of local parameters yields an isomorphism $F^{\times} \simeq \mathbb{Z}^{\oplus n} \oplus \mathbb{F}_{q}^{\times} \oplus V_{F}$, where $\mathbb{Z}^{\oplus n}$ corresponds to the cyclic groups generated by $t_{i}$.

For other details see [43] and articles of [14].

4.2. Topology on higher local fields. It is natural to expect the compatibility of theories of a complete discrete valuation field and its residue field. Thus, most interesting topologies of an $n$-dimensional local field have to take into account topologies of its residue field $k_{n-1}$ which is $(n-1)$-dimensional. Also, for many issues including class field theory, sequential continuity is more important than continuity. This is a hidden phenomenon for classical local fields, where continuity is the same as sequential continuity.

Definition 4.3. Topology on the additive group. Define the following topology $\lambda$ on the additive group of $F$ by induction on dimension. For 1-dimensional local fields this is just the standard topology of local fields with finite residue fields. For local parameters $t_{n}, \ldots, t_{1}$ of $F$ consider their residues $\bar{t}_{n-1}, \ldots, \bar{t}_{1} \in k_{n-1}$. Then the field $k_{n-1}$ is isomorphic to the field of formal power series $\mathbb{F}_{q}\left(\left(\bar{t}_{1}\right)\right) \ldots\left(\left(\bar{t}_{n-1}\right)\right)$. Define a coefficient field $E$ of $k_{n-1}$ in $F$ as the image of the field homomorphism which sends such a formal power series in $k_{n-1}$ to the formal power series in $F$ that is obtained by replacing $\bar{t}_{i}$ with $t_{i}$. Then $E=\mathbb{F}_{q}\left(\left(t_{1}\right)\right) \ldots\left(\left(t_{n-1}\right)\right), F=E\left(\left(t_{n}\right)\right)$.

Given the topology $\lambda$ on the additive group $k_{n-1}$, introduce the following translation invariant topology on the additive group $F$. An element $\alpha \in F$ is said to be a limit of a sequence of elements $\alpha_{j} \in F$ if and only if given any convergent (in the topology of complete discrete valuation field) series $\alpha_{j}=\sum_{i} \theta_{j, i} t_{n}^{i}, \alpha=\sum_{i} \theta_{i} t_{n}^{i}$ with $\theta_{*} \in E$ the following property holds: for every $i_{0}$ and for every set of neighbourhoods $U_{i}, \mathbb{Z} \ni i<i_{0}$, of zero in $k_{n-1}$, for almost all $j$ the residue of $\theta_{j, i}-\theta_{i}$ belongs to $U_{i}$ for all $i<i_{0}$. A subset $U$ in $F$ is called open if and only if it is sequentially open, i.e. for every $\alpha \in U$ and every sequence $\alpha_{j} \in F$ having $\alpha$ as a limit almost all $\alpha_{j}$ belong to $U$. This determines the translation invariant topology $\lambda$ on $F$.

By induction on dimension one verifies that a limit is uniquely determined, each Cauchy sequence with respect to the topology $\lambda$ converges in $F$, the limit of the sum of two convergent sequences is the sum of their limits. The Hausdorff topology $\lambda$ does not depend on the choice of local parameters. 
Given a topological space with its topology $\tau$, the finest topology on the underlying set which has the same sequences of fundamental sequences is called the sequential saturation of $\tau$. If the sequential saturation of a topology is equivalent to the topology $\tau$, the topology $\tau$ is called sequentially saturated. The topology $\lambda$ is sequentially saturated.

Definition 4.4. Call a subset $X$ of positive elements of $(\mathbb{Z})^{n}$ admissible if (a) for every $1<m \leqslant n$ and every $\left(i_{m}, \ldots, i_{n}\right)$ there is an integer $j\left(i_{m}, \ldots, i_{n}\right)$ such that $\left(i_{1}, \ldots, i_{m}, \ldots, i_{n}\right) \in X$ implies $i_{m-1} \geqslant j\left(i_{m}, \ldots, i_{n}\right)$, (b) there is $j$ such that all $\left(i_{1}, \ldots, i_{n}\right) \in X$ implies $i_{n} \geqslant j$.

Every element $\alpha \in F$ can be expanded into a convergent with respect to $\lambda$ series:

$$
\alpha=\sum \theta_{i_{n}, \ldots, i_{1}} t_{n}^{i_{n}} \ldots t_{1}^{i_{1}}, \quad \theta_{i_{n}, \ldots, i_{1}} \in \mathbb{F}_{q}
$$

with admissible set $\left\{\left(i_{1}, \ldots, i_{n}\right): \theta_{i_{n}, \ldots, i_{1}} \neq 0\right\}$, see e.g. [44].

Remark 4.5. If a subset $A=\left\{\sum a_{i} t_{n}^{i} \in F: a_{i} \in S_{i}\right\}$ with subsets $S_{i}$ of $E$ such that $S_{i}=E$ for all sufficiently large $i$, is open, then the isomorphic residue map images of $S_{i}$ are open in $k_{n-1}$. The paper [33, sect.1 Def.2] defined a translation invariant topology on the additive group $F$ such that the subsets $A$ form its basis of neighbourhoods of zero. Denote this topology by $\lambda_{0}$. Compare $\lambda_{0}$ with the direct limit of the topologies on $t_{i} \mathscr{O}_{F}$ endowed with the inverse limit of the topologies of $t_{i} \mathscr{O}_{F} / t_{i+j} \mathscr{O}_{F} \simeq k_{n-1}^{\oplus j}$.

A sequence of elements in $F$ converges to $x \in F$ with respect to $\lambda$ if and only if it converges with respect to the topology of $\lambda_{0}$. The topology $\lambda$ is the sequential saturation of $\lambda_{0}$.

By induction on $n$ one immediately checks that a subgroup of $F$ is open in $\lambda$ if and only if it is open in $\lambda_{0}$.

With respect to the topology $\lambda_{0}$ we have the same convergent series presentation of elements of $F$ as the one for the topology $\lambda$.

For various reasonable additive functors, such as the Witt functor $W_{m}$, we have similar results.

For $n \geqslant 2$, the topology $\lambda$ on the additive group is strictly finer than $\lambda_{0}$. For example, the set $D=F \backslash$ $\left\{t_{2}^{a} t_{1}^{-c}+t_{2}^{-a} t_{1}^{c}: a, c \geqslant 1\right\}$ in $F=\mathbb{F}_{p}\left(\left(t_{1}\right)\right)\left(\left(t_{2}\right)\right)$ is open in $\lambda$ but not in $\lambda_{0}$. Indeed, (a) for each convergent sequence $x_{j} \rightarrow x \in D$ almost all $x_{j}$ belong to $D$; (b) if for some open subsets $U_{i}$ in the additive group of $\mathbb{F}_{p}\left(\left(t_{1}\right)\right)$ such that $U_{i}=\mathbb{F}_{p}\left(\left(t_{1}\right)\right)$ for $i \geqslant a$ the open set $\left\{x=\sum a_{i} t_{2}^{i}: x \in F, a_{i} \in U_{i}\right\}$ in the topology $\lambda_{0}$ were contained in $D$, then for any positive $c$ such that $t_{1}^{c} \in U_{-a}$ we would have $t_{2}^{a} t_{1}^{-c}+t_{2}^{-a} t_{1}^{c} \in D$, a contradiction.

For various additive aspects one can work with $\lambda_{0}$ on $F$ and $\lambda$ is not required. In particular, the current topology on additive Witt groups in this paper is defined as the inverse limit topology. But if we work with the sequential saturation of the current topology, all the results remain true, since the study of characters of such groups and their quotients involves cosets of subgroups only, and the set of open subgroups remains the same when one passes to the sequential saturation.

The additive group $F$ is a topological group with respect to $\lambda_{0}$. The group $F$ is not a topological group for $n \geqslant 2$ with respect to $\lambda$. For example, any subset $D^{\prime}$ containing zero and such that $D^{\prime}+D^{\prime} \subset D$ is not open with respect to $\lambda$.

The topology $\lambda$ is of much more importance for the multiplicative group and Milnor $K$-groups.

Definition 4.6. Multiplicative topology. Define the topology $\lambda^{\times}$on $F^{\times}$as the sequential saturation of the product topology of the induced from $F$ topology on the group of principal units $V_{F}$, the discrete topologies on the cyclic groups generated by $t_{i}$ and the cyclic group $\mathbb{F}_{q}^{\times}$. 
It is easy to check that the multiplication in $F^{\times}$is sequentially continuous. Similarly to the one-dimensional case, every element $\alpha \in F^{\times}$can be expanded into a convergent with respect to $\lambda^{\times}$product:

$$
\alpha=t_{n}^{a_{n}} \ldots t_{1}^{a_{1}} \theta \prod\left(1+\theta_{i_{n}, \ldots, i_{1}} t_{n}^{i_{n}} \ldots t_{1}^{i_{1}}\right), \quad \theta \in \mathbb{F}_{q}^{\times}, \theta_{i_{n}, \ldots, i_{1}} \in \mathbb{F}_{q}
$$

with admissible set $\left\{\left(i_{1}, \ldots, i_{n}\right): \theta_{i_{n}, \ldots, i_{1}} \neq 0\right\}$ as in Definition 4.4, see e.g. [44].

By induction on dimension one checks that for every sequence $a_{i} \in V_{F}$, the sequence $a_{i}^{p^{i}}$ converges to 1 , subgroups $V_{F}$ and $F^{\times^{m}}$ are closed subgroups of $F^{\times}$, the intersection of a decreasing sequence of non-empty closed subsets is non-empty.

Remark 4.7. For a 2-dimensional local field its multiplicative group $F^{\times}$is a topological group with respect to $\lambda^{\times}$and it has a countable base of open subgroups. In the case of an $n$-dimensional local fields field with $n \geqslant 3$ both assertions do not hold. For example, for a subset $D^{\prime}$ of $1+\mathscr{O}_{L} t_{3}$ containing 1 , if $D^{\prime} \cdot D^{\prime} \subset 1+D t_{3}+\mathscr{O}_{L} t_{3}^{2}$ for $L=F\left(\left(t_{3}\right)\right)$ with $D$ as above, then $D^{\prime}$ is not open in $1+\mathscr{O}_{L} t_{3}$.

Remark 4.8. The paper [33] introduced a translation invariant topology $\lambda_{0}^{\times}$on $F^{\times}$as the product topology of the following topologies: the topology of principal units $V_{F}$ induced from the topology $\lambda_{0}$ (see Remark 4.5) and the discrete topologies on the cyclic groups generated by $t_{i}$ and the cyclic group $\mathbb{F}_{q}^{\times}$.

Note that if $n \geqslant 2$, then the product of two ( $\lambda$ - or $\lambda_{0^{-}}$) open subgroups of $F$ equals $F$ : for an open subgroup $R=\left\{\sum a_{i} t_{n}^{i} \in F: a_{i} \in S_{i}\right\}$ with open (hence non-empty) subgroups $S_{i}$ of $S$ the product $R R=F$.

So for $n \geqslant 3$ the group $\left(1+t_{n} R+t_{n}^{2} R\right)\left(1+t_{n} R+t_{n}^{2} R\right)\left(1+t_{n}^{3} O_{F}\right)$ contains $1+t_{n}^{2} a b$ for any $a, b \in R$, hence it includes $1+t_{n}^{2} O_{F}$. Therefore, for $n \geqslant 3$, for each open subgroup $B$ in $F^{\times}$with respect to the topology $\lambda_{0}^{\times}$ we have $1+t_{n}^{2} O_{F} \subset\left(1+t_{n}^{3} O_{F}\right) B$. This is clearly an unsatisfactory situation if one aims to study principal units topologically. In particular, the subgroup $1+t_{n} R+t_{n}^{2} R+t_{n}^{3} O_{F}$ is not $\lambda_{0}^{\times}$-open but it is $\lambda^{\times}$-open, so the two topologies are different even at the level of subgroups.

For other details see [43], [10] and articles of [14].

\subsection{Milnor and topological Milnor $K$-groups of higher local fields.}

Definition 4.9. Let $\lambda^{m}$ be the finest topology on the Milnor $K$-group $K_{m}(F)$ such that the subtraction in $K_{m}(F)$ and the symbol homomorphism

$$
\left(F^{\times}\right)^{\oplus m} \longrightarrow K_{m}(F)
$$

are sequentially continuous. Then $\lambda^{m}$ is a translation invariant topology which is sequentially saturated.

Denote by $\Lambda_{m}(F)$ the intersection of all open neighbourhoods of $0 \in K_{m}(F)$ with respect to $\lambda^{m}$. A constant sequence $\alpha_{i}=\alpha$ converges to 0 iff $\alpha \in \Lambda_{m}(F)$. Hence $\Lambda_{m}(F)$ is a closed subgroup of $K_{m}(F)$. Set

$$
K_{m}^{\text {top }}(F):=K_{m}(F) / \Lambda_{m}(F)
$$

with the quotient topology of $\lambda^{m}$ which we will denote by the same notation.

Remark 4.10. To the best of our knowledge, the Quillen $K$-groups, unless they coincide with the Milnor $K$-groups, are not really useful for class field theory purposes.

We have $\lambda^{1}=\lambda^{\times}$and $K_{1}^{\text {top }}(F)=F^{\times}$.

Denote by $V K_{m}(F)$ the subgroup of $K_{m}(F)$ generated by $\left\{V_{F}\right\} \cdot K_{m-1}(F)$. It is easy to see, using the first isomorphism of the following Lemma, that $\Lambda_{m}(F) \subset V K_{m}(F)$. Denote by $V K_{m}^{\text {top }}(F)$ the image of $V K_{m}(F)$ in $K_{m}^{\mathrm{top}}(F)$, endow it with the induced topology.

Endow $K_{m}(F) / p^{r} K_{m}(F), K_{m}^{\text {top }}(F) / p^{r} K_{m}^{\text {top }}(F), V K_{m}^{\text {top }}(F) / p^{r} V K_{m}^{\text {top }}(F)$ with the induced topologies.

Recall the well known 
Lemma 4.11. We have algebraic isomorphisms

$$
K_{n}(F) \simeq \mathbb{Z} \oplus\left(\mathbb{F}_{q}^{\times}\right)^{\oplus n} \oplus V K_{n}(F), \quad K_{n}^{\text {top }}(F) \simeq \mathbb{Z} \oplus\left(\mathbb{F}_{q}^{\times}\right)^{\oplus n} \oplus V K_{n}^{\text {top }}(F),
$$

where the first infinite cyclic group is generated by $\left\{t_{1}, \ldots, t_{n}\right\}$ where $t_{n}, \ldots, t_{1}$ are local parameters of $F$, and the jth copy of $\mathbb{F}_{q}^{\times}$corresponds to $\left\{t_{1}, \ldots, \theta, \ldots t_{n}\right\}$ with $\theta \in \mathbb{F}_{q}^{\times}$instead of $t_{j}$.

Proof. Use $K_{2}\left(\mathbb{F}_{q}\right)=0$ and $V_{F}=V_{F}^{q-1}$.

$\mathbb{Z}_{p}$ acts on the group $V K_{n}^{\text {top }}(F)$ continuously.

Proposition 4.12. Every element $x \in V K_{n}^{\mathrm{top}}(F)$ is the convergent (with respect to $\lambda^{n}$ ) series

$$
\sum a_{i, S, J}\left\{1+\theta_{i} t_{n}^{s_{n}} \cdots t_{1}^{s_{1}}, t_{j_{1}}, \cdots, t_{j_{n-1}}\right\}
$$

where $a_{i, S, J} \in \mathbb{Z}_{p}, \theta_{i}$ are elements of a fixed basis $B$ of $\mathbb{F}_{q}$ over $\mathbb{F}_{p}$ and $\theta_{1}=1,\left\{S=\left(s_{1}, \ldots, s_{n}\right): a_{i, S, j} \neq 0\right\}$ is an admissible set, $1 \leqslant j_{1}<\cdots<j_{n-1} \leqslant n, p$ does not divide at least one of $s_{1}, \ldots, s_{n}, \min \left\{v: p \nmid s_{v}\right\} \notin J=$ $\left\{j_{1}, \cdots, j_{n-1}\right\}$.

Proof. The following formula is straightforward:

$$
\{a, b\}=\{1-a, 1+(a-1) b\}-\left\{1+(a-1)(b-1) a^{-1}, b\right\} .
$$

If $a, b$ are in $V_{F}$, then the unit $1+(a-1)(b-1) a^{-1}$ is nearer to 1 than $a, b$ are.

Now for $1+\beta \in V_{F}$ and $\theta \in \mathbb{F}_{q}^{\times}$, we get

$$
\left\{1-\theta t_{n}^{s_{n}} \ldots t_{1}^{s_{1}}, 1+\beta\right\}=\left\{\theta t_{n}^{s_{n}} \ldots t_{1}^{s_{1}}, 1-\theta t_{n}^{s_{n}} \ldots t_{1}^{s_{1}}(1+\beta)\right\}-\left\{1-\theta t_{n}^{s_{n}} \ldots t_{1}^{s_{1}} \beta\left(1-\theta t_{n}^{i_{n}} \ldots t_{1}^{i_{1}}\right)^{-1}, 1+\beta\right\} .
$$

The first symbol of the right hand side can be written as the sum of symbols $\left\{t_{i}, \rho_{i}\right\}$ with principal units $\rho_{i}$ which sequentially continuously depend on $1-\theta t_{n}^{s_{n}} \ldots t_{1}^{s_{1}}$ and $1+\beta$, and the first element of the second symbol is closer to 1 than $1-\theta t_{n}^{s_{n}} \ldots t_{1}^{s_{1}}$ is. Using the topological convergence we obtain the result, without the restriction on indices. This restriction follows from

$$
0=\left\{1+\theta t_{n}^{s_{n}} \cdots t_{1}^{s_{1}},-\theta t_{n}^{s_{n}} \cdots t_{1}^{s_{1}}\right\}=\sum_{i=1}^{n}\left\{1+\theta t_{n}^{s_{n}} \cdots t_{1}^{s_{1}}, t_{i}\right\}+\left\{1+\theta t_{n}^{s_{n}} \cdots t_{1}^{s_{1}},-\theta\right\}=\sum_{i=1}^{n}\left\{1+\theta t_{n}^{s_{n}} \cdots t_{1}^{s_{1}}, t_{i}\right\},
$$

since $V_{F}=V_{F}^{q-1}$.

Let $\chi$ be a continuous character of $K_{n}^{\text {top }}(F)$, i.e. a homomorphism to the unit circle which is sequentially continuous. Find a positive integer $l(n)$ such that $\left\{1+t_{n}^{l(n)} \mathscr{O}_{F}, t_{j_{1}}, \ldots, t_{j_{n-1}}\right\}$ is in the kernel of $\chi$ for every $1 \leqslant j_{1}<\cdots<j_{n-1} \leqslant n$. Find a positive integer $r(n)$ such that $\left(1+t_{n} \mathscr{O}_{F}\right)^{r(n)}<1+t_{n}^{l(n)} \mathscr{O}_{F}$. Hence $\chi^{r(n)}$ is trivial on the subgroup generated by $1+t_{n} \mathscr{O}_{F}$. Continue with $\left\{1+t_{n-1}^{l(n-1)} \mathscr{O}_{E}, t_{j_{1}}, \ldots, t_{j_{n-1}}\right\}$, where $E$ is the coefficient field of $k_{n-1}$ in $F$, etc. Thus, every continuous character of $K_{n}^{\text {top }}(F)$ is of finite order.

For other details see [44], [10] and articles of [14].

4.4. Generalised Artin-Schreier-Witt pairing. Define the current topologies on the groups $W(F), W_{m}(F)$ and $W_{m}(F) / \wp\left(W_{m}(F)\right)$ as the induced from the inverse limit topology on the product of countably many copies of $F$. All the properties of Definition 3.12 hold. Alternatively, one can work with their sequential saturation, since the class of open subgroups does not get larger, see Remark 4.5.

To obtain a description of $V_{n}^{\text {top }}(F)$ in terms of its $\mathbb{Z}_{p}$-topological generators, we use a generalisation of the Artin-Schreier-Witt pairing for one-dimensional local fields (3.25) to higher local fields of positive characteristic. It seems to first appear in [33]. 
Put $O_{0}=W\left(\mathbb{F}_{q}\right)$. For $\alpha_{1}, \ldots, \alpha_{n} \in F^{\times}$, and a Witt vector $\beta=\left(\beta_{0}, \ldots, \beta_{m}\right) \in W_{m}(F)$ put

$$
\left(\alpha_{1}, \ldots, \alpha_{n}, \beta\right)_{m}=\operatorname{Tr}_{\mathbb{F}_{q} / \mathbb{F}_{p}}(\gamma)
$$

where the $i$ th ghost component $\gamma^{(i)} \in O_{0}\left(\left(X_{1}\right)\right) \ldots\left(\left(X_{n}\right)\right)$ of $\gamma$ is defined as $\operatorname{res}_{\mathbb{F}_{q}}\left(\beta^{(i)} d \alpha_{1} / \alpha_{1} \wedge \cdots \wedge d \alpha_{n} / \alpha_{n}\right)$ where $\beta^{(i)} \in O_{0}\left(\left(X_{1}\right)\right) \ldots\left(\left(X_{n}\right)\right)$ is the $i$ th ghost component of $\underline{\beta}, \underline{\alpha}$ is any element of $O_{0}\left(\left(X_{1}\right)\right) \ldots\left(\left(X_{n}\right)\right)$ which surjects to $\alpha$ with respect to the canonical homomorphism to $\bar{F}, \underline{\beta}$ is any element of $\left.W_{m}\left(O_{0}\left(\left(X_{1}\right)\right)\right) \ldots\left(\left(X_{n}\right)\right)\right)$ which surjects to $\beta$.

Thus we get the pairing

$$
F^{\times} / F^{\times p^{m}} \times \cdots \times F^{\times} / F^{\times p^{m}} \times W_{m}(F) \longrightarrow W_{m}\left(\mathbb{F}_{p}\right) .
$$

This is a map that is sequentially continuous in each of the variables and symbolic in the first $n$ variables, hence it induces a continuous in each variable pairing

$$
(,)_{m}: K_{n}^{\mathrm{top}}(F) / p^{m} K_{n}^{\mathrm{top}}(F) \times W_{m}(F) \longrightarrow W_{m}\left(\mathbb{F}_{p}\right) .
$$

It is known that this pairing is well defined and the properties (A1), (A2), (A3) of Definition 3.14 are satisfied, see e.g. [33]. Thus we get the pairing

$$
\langle,\rangle_{m}: K_{n}^{\mathrm{top}}(F) / p^{m} K_{n}^{\mathrm{top}}(F) \times W_{m}(F) / \wp\left(W_{m}(F)\right) \longrightarrow W_{m}\left(\mathbb{F}_{p}\right) .
$$

Choose elements $\eta_{j} \in \mathbb{F}_{q}^{\times}$which form the dual basis of $\mathbb{F}_{q}$ over $\mathbb{F}_{p}$ with respect to the basis $B$ in Proposition 4.12. It is easy to show, similarly to the one-dimensional case, see Example 3.25, that the group $F / \wp(F)$ has topological generators $\eta_{1}$ and $\eta_{j} t_{1}^{r_{1}} \cdots t_{n}^{r_{n}}$ where $\left(r_{1}, \cdots, r_{n}\right)<0$ and $\operatorname{gcd}\left(p, r_{1}, \cdots, r_{n}\right)=1$. The use of just one element $\eta_{1}$ for $\left(r_{1}, \cdots, r_{n}\right)=0$ follows from the definitions.

Similarly to the one-dimensional case in 3.25 , to show the non-degeneracy of $\langle,\rangle_{m}$ we can use $\mathbb{Z} / p^{m} \mathbb{Z}$ topological generators of $K_{n}^{\mathrm{top}}(F) / p^{m} K_{n}^{\mathrm{top}}(F)$ described in Proposition 4.12, and $\mathbb{Z} / p^{m} \mathbb{Z}$-topological generators of $W_{m}(F) / \wp\left(W_{m}(F)\right.$ described above. Computing, we get

$$
\begin{aligned}
& \left\langle\left\{t_{1}, \cdots, t_{n}\right\}, \eta_{1}\right\rangle_{1}=1, \quad\left\langle\left\{1+\theta_{i} t_{n}^{s_{n}} \cdots t_{1}^{s_{1}}, t_{j_{1}}, \cdots, t_{j_{n-1}}\right\}, \eta_{1}\right\rangle_{1}=0, \quad\left\langle\left\{t_{1}, \cdots, t_{n}\right\}, \eta_{j} t_{1}^{r_{1}} \cdots t_{n}^{r_{n}}\right\rangle_{1}=0, \\
& \left\langle\left\{1+\theta_{i} t_{n}^{s_{n}} \cdots t_{1}^{s_{1}}, t_{j_{1}}, \cdots, t_{j_{n-1}}\right\}, \eta_{j} t_{1}^{r_{1}} \cdots t_{n}^{r_{n}}\right\rangle_{1}=0 \\
& \text { if }\left(s_{1}, \cdots, s_{n}\right)+\left(r_{1}, \cdots, r_{n}\right)>0 \text { or }\left(s_{1}, \cdots, s_{n}\right)+\left(r_{1}, \cdots, r_{n}\right)=0 \text { and } i \neq j, \\
& \left\langle\left\{1+\theta_{i} t_{n}^{s_{n}} \cdots t_{1}^{s_{1}}, t_{j_{1}}, \cdots, t_{j_{n-1}}\right\}, \eta_{j} t_{1}^{r_{1}} \cdots t_{n}^{r_{n}}\right\rangle_{1}= \pm 1 \text { if }\left(s_{1}, \cdots, s_{n}\right)+\left(r_{1}, \cdots, r_{n}\right)=0 \text { and } i=j .
\end{aligned}
$$

These equalities allow to use Remark 3.24 in order to show that the two sets of topological generators are topological bases of $V K_{n}^{\mathrm{top}}(F) / p V K_{n}^{\mathrm{top}}(F)$ and of $F / \wp(F)$, and to deduce that the pairing $\langle,\rangle_{1}$ is nondegenerate.

It is not difficult to prove the non-degeneracy of $\langle,\rangle_{m}$ directly, using properties of Witt vectors, and in particular to deduce that the elements $\left(\eta_{1}, 0, \ldots\right),\left(\eta_{j} t_{1}^{r_{1}} \cdots t_{n}^{r_{n}}, 0, \ldots\right)$, where $\left(r_{1}, \cdots, r_{n}\right)<0$ and $\operatorname{gcd}\left(p, r_{1}, \cdots, r_{n}\right)=$ 1, form a topological basis of $W_{m}(F) / \wp\left(W_{m}(F)\right)$, using arguments similarly to the arguments in [27], [35], [33], see also [42]. Alternatively, property (A5) in subsection 3.3 shows that the pairing $\langle,\rangle_{m}$ is non-degenerate and then using Remark 3.24 one immediately checks that the topological generators in Proposition 4.12 form a topological basis of $V K_{n}^{\text {top }}(F) / p^{m} V K_{n}^{\text {top }}(F)$.

If $\alpha \in \cap_{m \geqslant 1} p^{m} V K_{n}^{\text {top }}(F)$ then $\alpha=\sum_{j_{1}<\cdots<j_{n-1}}\left\{\rho_{r, j_{1}, \ldots, j_{n-1}}^{p^{r}}, t_{j_{1}}, \cdots, t_{j_{n-1}}\right\}$ with principal units $\rho_{r, j_{1}, \ldots j_{n-1}}$. Hence $\cap_{m \geqslant 1} p^{m} V K_{n}^{\text {top }}(F) \subset \Lambda_{n}(F)$. Using Lemma 4.11 we obtain 
Proposition 4.13. The elements $\left\{1+\theta_{i} t_{n}^{s_{n}} \cdots t_{1}^{s_{1}}, t_{j_{1}}, \cdots, t_{j_{n-1}}\right\}$, where $\theta_{i}$ are elements of a fixed basis $B$ of $\mathbb{F}_{q}$ over $\mathbb{F}_{p}$ and $\theta_{1}=1,1 \leqslant j_{1}<\cdots<j_{n-1} \leqslant n, \operatorname{gcd}\left(p, s_{1}, \ldots, s_{n}\right)=1, \min \left\{v: p \nmid s_{v}\right\} \notin\left\{j_{1}, \cdots, j_{n-1}\right\}$, form a topological $\mathbb{Z}_{p}$-basis of $V K_{n}^{\text {top }}(F)$.

These elements and the elements $\left\{t_{1}, \ldots, t_{n}\right\},\left\{t_{1}, \ldots, \theta_{i}, \ldots t_{n}\right\}$ with $\theta_{i} \in \mathbb{F}_{q}^{\times}$instead of $t_{j}, 1 \leqslant j \leqslant n$, form a topological $\mathbb{Z}_{p}$-basis of $K_{n}^{\mathrm{top}}(F)$.

In particular, $K_{n}^{\mathrm{top}}(F)$ has no nontrivial p-torsion.

As a side remark, recall that the general theorem of Izhboldin, whose proof is of different nature than the proof of the previous proposition, tells that the Milnor $K$-group $K_{n}(k)$ has no nontrivial $p$-torsion if $k$ is a field of characteristic $p$.

We now prove

Proposition 4.14. The pairing $\langle,\rangle_{1}$ is algebraically perfect.

Proof. The proof follows the proof of [3, Ch.3 sect.4 Prop.5]. We prove that $\langle,\rangle_{1}$ is algebraically perfect on the right, i.e. $\xi_{1}$ of Definition 3.3 is surjective. The proof that $\zeta_{1}$ is surjective goes in the similar way.

Let $\chi$ be a continuous character or order $p$ of $K_{n}^{\text {top }}(F) / p K_{n}^{\text {top }}(F)$. Denote its kernel by $C$, this is an open and closed subgroup of index $p$ of $K_{n}^{\text {top }}(F) / p K_{n}^{\text {top }}(F)$. We will work with the set $Z$ of elements $\left\{t_{1}, \ldots, t_{n}\right\}$ and $\left\{1+\theta t_{n}^{s_{n}} \cdots t_{1}^{s_{1}}, t_{j_{1}}, \cdots, t_{j_{n-1}}\right\}, \theta \in \mathbb{F}_{q}^{\times}$. Associating $\left(s_{n}, \ldots, s_{1}\right)$ to the element $\left\{1+\theta t_{n}^{s_{n}} \cdots t_{1}^{s_{1}}, t_{j_{1}}, \cdots, t_{j_{n-1}}\right\}$ and $+\infty$ to the element $\left\{t_{1}, \ldots, t_{n}\right\}$, we have a total ordering on the set of these elements.

For each $x \in Z$ denote by $x^{\prime}$ the element of the set $\eta, \eta t_{1}^{r_{1}} \cdots t_{n}^{r_{n}}$ where $\left(r_{1}, \cdots, r_{n}\right)<0$ and $\operatorname{gcd}\left(p, r_{1}, \cdots, r_{n}\right)=$ $1, \eta \in \mathbb{F}_{q}^{\times}$, that satisfies the properties: $\left\langle x, x^{\prime}\right\rangle_{1}= \pm 1$ and $\left\langle y, x^{\prime}\right\rangle_{1}=0$ for all other elements $y \in Z, y>x$. For example, $\left\{t_{1}, \ldots, t_{n}\right\}^{\prime}=\eta_{1},\left\{1+\theta t_{n}^{s_{n}} \cdots t_{1}^{s_{1}}, t_{j_{1}}, \cdots, t_{j_{n-1}}\right\}^{\prime}=\eta t_{1}^{-s_{1}} \cdots t_{n}^{-s_{n}}$ with $\eta \in \mathbb{F}_{q}^{\times}, \operatorname{Tr}_{\mathbb{F}_{q} / \mathbb{F}_{p}}(\theta \eta)=1$.

Since $C$ is open, there is the largest $x \in Z$ that does not belong to $C$. If the order of $x$ is $\left(s_{n}, \ldots, s_{1}\right)<+\infty$, then the image $C_{1}$ of the subgroup $\left\{1+t_{n}^{s_{n}} \ldots t_{1}^{s_{1}+1} O_{F}\right\} K_{n-1}^{\text {top }}(F)$ in $K_{n}^{\text {top }}(F) / p K_{n}^{\text {top }}(F)$ is inside $C$. If the order of $x$ is $+\infty$, put $C_{1}=0$.

For every $y<x, y \in Z$, there is an $a_{y} \in \mathbb{Z} / p \mathbb{Z}$ such that $\hat{y}=y+a_{y} x \in C$. Then $\left\langle\hat{y}, z^{\prime}\right\rangle_{1}=0$ if $y>z$ and $= \pm 1$ if $y=z$. So we can find $b_{y} \in \mathbb{Z} / p \mathbb{Z}$ such that the element $f=x^{\prime}+\sum_{y<x} b_{y} y^{\prime}$, where $y$ runs through decreasing (in the sense of the ordering) elements of $Z$ and the series converges, has the property $\langle x, f\rangle_{1}= \pm 1$ and $\langle\hat{y}, f\rangle_{1}=0$ for all $y<x$. Note that $\left\langle C_{1}, f\right\rangle_{1}=0$.

For every $z \in C$ one can find its convergent series presentation as $z=\sum_{y<x} c_{y} \hat{y}+C_{1}$ with $c_{y} \in \mathbb{Z} / p \mathbb{Z}$. Hence $\langle C, f\rangle_{1}=0$ and $\langle x, f\rangle_{1}= \pm 1$. Therefore, $\chi=\xi_{1}(a f)$ for some non-zero $a \in \mathbb{Z} / p \mathbb{Z}$.

4.5. The group $\Lambda_{n}(F)$ and the norm map. The following theorem was proved in [10].

Theorem 4.15. Let $F$ be an n-dimensional local field. Then

$$
\Lambda_{n}(F)=\bigcap_{l \geqslant 1} l K_{n}(F) .
$$

Proof. One inclusion is straightforward from $\cap_{m \geqslant 1} p^{m} V K_{n}^{\text {top }}(F) \subset \Lambda_{n}(F)$ mentioned earlier. The prove the other inclusion we use the Bloch-Kato-Gabber theorem that claims that the differential symbol

$$
d_{F}: K_{n}(F) / p K_{n}(F) \longrightarrow \operatorname{ker}\left(\wp: \Omega_{F}^{n} \rightarrow \Omega_{F}^{n} / d \Omega_{F}^{n-1}\right), \quad\left\{a_{1}, \cdots, a_{n}\right\} \rightarrow \frac{d a_{1}}{a_{1}} \wedge \cdots \wedge \frac{d a_{n}}{a_{n}}
$$

is an isomorphism. Here $\wp: a d b_{1} / b_{1} \wedge \cdots \wedge d b_{n} / b_{n} \mapsto\left(a^{p}-a\right) d b_{1} / b_{1} \wedge \cdots \wedge d b_{n} / b_{n}+d \Omega_{F}^{n-1}$ is the Cartier operator. For a compact proof of this theorem see e.g. [14, Append. to Part I sect.2] 
Since $\Omega_{F}^{n}$ is a finite-dimensional vector space over $F / F^{p}$ and $d_{F}$ is sequentially continuous, the intersection of all neighbourhoods of zero in $\Omega_{F}^{n}$ with respect to the topology induced by $\lambda^{n}$ is trivial. Injectivity of the differential symbol $d_{F}$ now implies $\Lambda_{n}(F) \subset p K_{n}(F)$.

Since $K_{n}^{\text {top }}(F)$ does not have nontrivial $p$-torsion by Proposition 4.13, from $\Lambda_{n}(F) \subset p K_{n}(F)$ we deduce that $\Lambda_{n}(F)=p \Lambda_{n}(F)$. Since $V_{F}=V_{F}^{l}$ for every $l$ prime to $p$, we get the assertion of the theorem.

Due to this theorem we now know that $N_{L / F} \Lambda_{n}(L) \subset \Lambda_{n}(F)$. Indeed, $N_{L / F} \Lambda_{n}(L)=N_{L / F}\left(\cap_{l \geqslant 1} l K_{n}(L)\right) \subset$ $\cap_{l \geqslant 1} l K_{n}(F)=\Lambda_{n}(F)$. Define the norm map on $K_{n}^{\text {top }}$-groups as the induced one.

Remark 4.16. By looking at closed subgroups of finite index of $V K_{n}^{\mathrm{top}}(F)$ generated by all elements of its $\mathbb{Z}_{p}$-topological basis, except one of them $x$, and by $x^{p^{r}}$, we deduce that the intersection of closed subgroups of $V K_{n}^{\text {top }}(F) / p^{m} V K_{n}^{\text {top }}(F)$ is trivial and the intersection of closed subgroups of finite index in $K_{n}^{\text {top }}(F)$ is trivial.

Note that $V K_{n}^{\mathrm{top}}(F) / p^{m} V K_{n}^{\mathrm{top}}(F)$ are not compact for $n>1$. For instance, if $F=\mathbb{F}_{q}\left(\left(t_{1}\right)\right)\left(\left(t_{2}\right)\right)$, define $S_{r}$ as the closed subgroup of $V K_{2}^{\text {top }}(F) / p^{m} V K_{2}^{\text {top }}(F)$ topologically generated, in the sense of Proposition 4.12, by elements $\left\{1+t_{2} t_{1}^{-p i}, t_{1}\right\}$ with $i>r$. Then the sets $U_{r}=V K_{n}^{\text {top }}(F) / p^{m} V K_{n}^{\text {top }}(F) \backslash S_{r}$, for $r \geqslant 1$ form an open cover of $V K_{n}^{\text {top }}(F) / p^{m} V K_{n}^{\text {top }}(F)$ which does not have a finite subcover.

Hence the algebraic isomorphism $\Psi_{H, m}: K_{n}^{\text {top }}(F) / p^{m} K_{n}^{\text {top }}(F) \simeq G\left({ }_{m} F / F\right)$ of Remark 3.16 is not a homeomorphism for $n>1$.

Remark 4.17. The topology on $K_{m}(F)$ in [33] was defined as the finest topology in which the symbol map is continuous in each variable and the subtraction on $K_{m}(F)$ is sequentially continuous. In view of Remark 4.8 and the problems with the topology $\lambda_{0}^{\times}$on $F^{\times}$, following this definition produces the topology $\lambda_{0}^{m}$ on $K_{m}(F)$ which does not have enough closed subgroups. For example, for $n=3$ the subgroup of $V K_{3}(F)$ which is the preimage of the closed subgroup of $V K_{3}^{\text {top }}(F)$ generated by all the elements of its topological basis except $\left\{1+t_{3}, t_{1}, t_{2}\right\}$ is not closed with respect to the topology $\lambda_{0}^{3}$. But this group is the norm group of an appropriate abelian extension of $F$. So the topology $\lambda_{0}^{3}$ is not useful for the existence theorem.

Another way to define of the norm map on $V K_{n}^{\text {top }}$ was proposed in [33], using the usual norm map on Milnor $K_{2}$-groups, as the map $N^{\prime}$ adjacent with respect to all the pairings $\langle,\rangle_{m}$ to the homomorphisms $W_{m}(F) / \wp\left(W_{m}(F)\right) \rightarrow W_{m}(L) / \wp\left(W_{m}(L)\right)$ induced by the field embedding $F \rightarrow L$. However, no verification that the usual norm map sends $\Lambda_{2}(L)$ to $\Lambda_{2}(F)$ was done, neither the commutativity of the diagram of [33, sect. 3 Lemma3] for topological $K_{2}$-groups was checked. To fix this gap one can use the previous Theorem and define the norm map on topological Milnor $K$-groups as the induced by the norm map on Milnor $K$-groups. Alternatively, one can prove the sequential continuity of the usual norm map $N_{L / F}$ and deduce that it sends $\alpha \in \Lambda_{n}(L)$, with the associated constant sequence $(\alpha)$ converging to 0 , to an element of $\Lambda_{n}(F)$, see e.g. [4, Lemma 3.3].

The proof of [34, Prop.2] claimed that for $K_{n}^{\text {top }}$-groups of higher local fields and the pairing $\langle,\rangle_{m}$ there is an extension of Pontryagin duality, by referring to [21]. However, that paper does not imply such an extension. It is a non-trivial issue whether the sequences of ๑-character groups are exact. The exactness is equivalent to the property of all continuous (with respect to the current topology) characters of a closed subgroup to be extendable to continuous characters of the full group. In the terminology of [2], in order the sequences to be exact, the closed image of each term of the sequence in the next term needs to be a dually embedded subgroup of the next term.

4.6. Higher local class field theory in positive characteristic. The objects in the image of the $\mathscr{A}$-functor of Definition 3.14 are the topological $K_{n}^{\text {top }}$-groups. We have already checked that the properties of Definition 3.14 hold. 
Proposition 4.13 implies that $\cap_{m \geqslant 0} p^{m} K_{n}^{\text {top }}(F)$ equals the subgroup $\left\{\mathbb{F}_{q}^{\times}\right\} K_{n-1}^{\text {top }}(F)$ generated by elements of $\mathbb{F}_{q}^{\times}$in $K_{n}^{\mathrm{top}}(F)$.

Thus, all the conditions of Definition 3.14 are satisfied. Via the class field theory mechanism in the previous section we get the main theorems of the $p$-primary part of higher local class field theory in positive characteristic $p$. This presentation simplifies and straightens [33], [34].

To get the full class field theory for $F$, i.e. not only the $p$-primary part, one adds two very easy parts.

The first part is the purely unramified $\hat{\mathbb{Z}}$-part, i.e. $G\left(F \mathbb{F}_{q}^{\text {sep }} / F\right)$. Here the reciprocity homomorphism sends $\left\{t_{1}, \ldots, t_{n}\right\}$ to the Frobenius automorphism in $G\left(F \mathbb{F}_{q}^{\text {sep }} / F\right)$ and $\left\{\mathbb{F}_{q}^{\times}\right\} K_{n-1}^{\text {top }}(F)$ and $V K_{n}^{\text {top }}(F)$ are sent to the identity automorphism.

The second part is $F\left(\sqrt[q-1]{F^{\times}}\right) / F$. Note that the maximal abelian totally tamely ramified extension of $F$ is $F\left(\sqrt[q-1]{t_{n}}, \ldots, \sqrt[q-1]{t_{1}}\right)$ and it is a subextension of this Kummer extension. Using Kummer theory and the higher tame symbol (see e.g. [14, 6.4.2]), instead of Witt theory and the Artin-Schreier-Witt pairing, one gets the reciprocity homomorphism $K_{n}^{\text {top }}(F) \longrightarrow G\left(F\left(\sqrt[q-1]{F^{\times}}\right) / F\right)$. With respect to it, $\left\{t_{1}, \ldots, t_{n}\right\}$ is sent to the Frobenius automorphism on $F\left(\sqrt[q-1]{\mathbb{F}_{q}^{\times}}\right) / F,\left\{t_{1}, \ldots, \theta, \ldots t_{n}\right\}$, with $\theta \in \mathbb{F}_{q}^{\times}$instead of $t_{j}$, is sent to an automorphism $\sigma(\theta, j)$ which acts trivially on $F\left(\sqrt[q-1]{\mathbb{F}_{q}^{\times}}, \sqrt[q-1]{t_{n}}, \ldots, \sqrt[q-1]{t_{1}}\right)$ (without $\sqrt[q-1]{t_{j}}$ ) and $\sigma(\theta, j)(u)=u \xi$, where $u^{q-1}=t_{j}$ and $\xi \in \mathbb{F}_{q}^{\times}$is the value (equal to $\pm \boldsymbol{\theta}^{ \pm 1}$ ) of the higher tame symbol on $-\left\{t_{1}, \ldots, t_{n}, \theta\right\} \in K_{n+1}^{\mathrm{top}}(F)$, and $V K_{n}^{\mathrm{top}}(F)$ is sent to the identity automorphism.

These three reciprocity homomorphisms are compatible with each other. It is not difficult to show that the residue maps in the diagram are sequentially continuous, see e.g. [42]. Thus, one also obtains the compatibility of class field theory for $F$ and its residue field $E$, which is an $(n-1)$-dimensional local field: the diagram

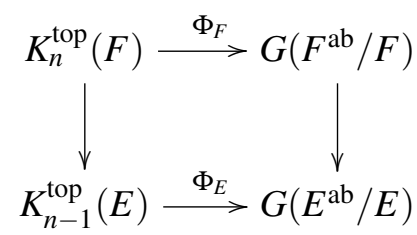

is commutative, where the left vertical arrow is induced by the boundary homomorphism in $K$-theory and the right vertical arrow comes from the valuation theory.

Thus, we get the full reciprocity homomorphism

$$
\Phi_{F}: K_{n}^{\mathrm{top}}(F) \longrightarrow G\left(F^{\mathrm{ab}} / F\right)
$$

with all the main properties similar to Theorems 3.19 and 3.20. For some of additional details see [42].

Remark 4.18. Similarly to Example 3.26, one can globalise this approach to the p-primary part of higher local class field theory to the $p$-primary part of class field theory of two-dimensional local-global fields of characteristic $p$. Two types of such fields are (a) the function field of the completion of the local ring of a smooth proper irreducible surface over a finite field at its irreducible curve, such fields are complete discrete valuations fields whose residue field is a one-dimensional global field, (b) the function field of the completion of the local ring of a smooth proper irreducible surface over a finite field at its closed point, such fields include finite extensions of the quotient fields of $\mathbb{F}_{q}[[X, Y]]$. Extending [33], [34] to the adelic setting, Syder developed various aspects of class field theories of such fields in [37]. In the statement of [37, Th. 4.1.2] and [37, Th. 5.1.2] 'topological isomorphism' should be replaced with 'algebraic isomorphism'. 


\section{REFERENCES}

[1] E. Artin, J.T. TATE, Class field theory, Benjamin 1968.

[2] M.J. Chasco, D. Dikranjan, E. Martín-Peinador, A survey of reflexivity of abelian topological groups, Topology and its applications, 159(2012) 2290-2309.

[3] I. Fesenko, Explicit constructions in local class field theory, PhD thesis, St Petersburg Univ. 1987.

[4] I. FESENKo, Class field theory of multidimensional local fields of characteristic zero, with residue field of positive characteristic, Algebra i Analiz (1991); English transl. in St. Petersburg Math. 3(1992) 649-678; Multidimensional local class field theory II, Algebra i Analiz (1991); English transl. in St. Petersburg Math. J. 3(1992), 1103-1126.

[5] I. FeSEnKo, Local class field theory: perfect residue field case, Izvest. Russ. Acad. Nauk. Ser. Mat. 1993; English transl. in Russ. Acad. Scienc. Izvest. Math. 43 (1994) 65-81.

[6] I. FesenKo, Abelian local p-class field theory, Math. Annal. 301 (1995) 561-586.

[7] I. FesenKo, On general local reciprocity maps, J. Reine Angew. Math. 473 (1996) 207-222.

[8] I. Fesenko, Noncommutative local reciprocity maps, In Class Field Theory - Its Centenary and Prospects, Adv. Studies in Pure Math., vol. 30, 63-78, Math. Soc. Japan 2001; available from https ://www . maths .nottingham. ac.uk/personal/ibf/ncr. pdf.

[9] I. FESENKO, On the image of noncommutative reciprocity map, Homology, Homotopy and Applications, 7 (2005) 53-62; available from https://www. maths. nottingham.ac.uk/personal/ibf/imn.pdf.

[10] I. Fesenko, Sequential topologies and quotients of Milnor K-groups of higher local fields, Algebra i Analiz, 2001; English transl. in St. Petersburg Math. J. 13 (2002), 485-501; available from https://www.maths.nottingham.ac.uk/personal/ $\mathrm{ibf} / \mathrm{stqk} \cdot \mathrm{pdf}$.

[11] I. Fesenko, Parshin's higher local class field theory in characteristic $p$, In Invitation to higher local fields, I. Fesenko and M. Kurihara (eds.), Geometry and Topology Monographs 3(2000) 75-79; available from https://msp. org/gtm/2000/03/.

[12] I. FesEnKo, Analysis on arithmetic schemes. II, J. K-theory, 5(2010) 437-557.

[13] I. FESENKo, Class field theory guidance and three fundamental developments in arithmetic of elliptic curves, preprint 2018; available from https://www.maths.nottingham.ac.uk/personal/ibf/232.pdf.

[14] I. Fesenko, M. Kurihara (EDS.), Invitation to higher local fields, Geometry and Topology Monographs 3(2000); available from https://www.maths.nottingham.ac.uk/personal/ibf/volume.html.

[15] I.B. Fesenko, S.V. Vostokov, Local fields and their extensions, 2nd extended ed., Amer. Math. Soc., 2002, available from https://www. maths.nottingham.ac.uk/personal/ibf/book/book.html

[16] K.I. IKedA, E. Serbest, Fesenko reciprocity map, St. Petersburg Math. J. 20 (2008) 407-445.

[17] K.I. IKedA, E. SERbeSt, Generalized Fesenko reciprocity map, St. Petersburg Math. J. 20 (2008) 593-624.

[18] K.I. IKedA, E. SERBeSt, Non-abelian local reciprocity law, Manuscripta Math. 132 (2010) 19-49.

[19] E. InABA, On matrix equations for Galois extensions of fields of characteristic p, Nat. Sc. Report, Ochanomizu Univ. 12(1961) $26-36$.

[20] E. INABA, On generalized Artin-Schreier equations, Nat. Sc. Report, Ochanomizu Univ. 13(1962) 1-13.

[21] S. KAPlan, Extensions of the Pontryagin duality, II: Direct and inverse limits, Duke Math. J. 17(1950) 419-435.

[22] K.KAто, A generalization of local class field theory by using K-groups. I, J. Fac. Sci. Univ. Tokyo Sect. IA Math. 26 (1979) 303-376; II, J. Fac. Sci. Univ. Tokyo Sect. IA Math. 27 (1980) 603-683.

[23] K.KATO, Galois cohomology of complete discrete valuation fields, Algebraic $K$-theory, Part II (Oberwolfach, 1980), Lecture Notes in Math. 967, Springer, 1982, pp. 215-238.

[24] K. Kato And S. Saito, Two-dimensional class field theory, In Galois groups and their representations, Adv. Stud. Pure Math., vol. 2, North-Holland, Amsterdam, 1983, pp. 103-152.

[25] Y. KAWADA, Class formations, In 1969 Number Theory Institute, Proc. Sympos. Pure Math. 20(1971) 96-114.

[26] Y. KAWADA, Class formations. I, Duke Math. J. 22(1955) 165-177.

[27] Y. Kawada, I. Satake, Class formations. II, J. Fac. Sci. Univ. Tokyo 7(1955) 453-490.

[28] Y. KoYA, A generalization of class formation by using hypercohomology, Invent. Math. 101(1990) 705-715.

[29] S. LANG, Algebra, Addison-Wesley, Reading, MA, 1965.

[30] Sh. Mochizuki, Topics in absolute anabelian geometry III: Global reconstruction algorithms, J. Math. Sci. Univ. Tokyo 22 (2015) 939-1156, available with comments from http://www.kurims.kyoto-u.ac.jp/ motizuki/papers-english . html 
[31] J. NEUKIRCH, Class field theory, Springer 1986.

[32] J. NEUKIRCH, Algebraic number theory, Springer 1999.

[33] A.N. PARShin, Local class field theory, Trudy Mat. Inst. Steklov (1985); English transl. in Proc. Steklov Inst. Math. 1985, issue $3,157-185$.

[34] A.N. PARShin, Galois cohomology and Brauer group of local fields, Trudy Mat. Inst. Steklov (1990); English transl. in Proc. Steklov Inst. Math. 1991, issue 4, 191-201.

[35] K. SeKiguchi, Class field theory of $p$-extensions over a formal power series field with a $p$-quasifinite coefficient field, Tokyo $J$. Math. 6(1983) 167-190.

[36] M. SPIESS, Class formations and higher-dimensional local class field theory, J. Number Theory 62(1997) $273-283$.

[37] K. Syder, Two-dimensional local-global class field theory in positive characteristic, PhD Thesis, Univ. Nottingham, 2014; available from https://arxiv.org/abs/1403.6747.

[38] P. WeBB, A guide to Mackey functors, available from http://web.math.rochester.edu/people/faculty/doug/ otherpapers/WebbMF.pdf

[39] A. WeIL, Basic number theory, Springer 1967.

[40] E. WITT, Konstruktion von galoisschen Körper der Charakteristik $p$ zu vorgegebener Gruppe der Ordnung $p^{f}$, J. Reine Angew. Math. 174(1936) 237-245.

[41] E. WitT, Der Existenzsatz für abelschen Funktionenkörper, J. Reine Anwew. Math. 173(1935) 43-51; Zyklische Körper und Algebren der Charakteristik $p$ von Grade $p^{n}$, J. Reine Anwew. Math. 176(1936) 126-140.

[42] S. H. Yoon, Explicit class field theory: one-dimensional and higher-dimensional, PhD thesis, Univ. Nottingham, 2017.

[43] I. Zhukov, A. Madunts, Multi-dimensional complete fields: topology and other basic constructions, In Trudy S.-Peterb. Mat. Obshch. (1995); English translation in Amer. Math. Soc. Transl. (Ser. 2) 165 (1995), 1-34.

[44] I. ZHUKOV, Milnor and topological K-groups of higher-dimensional complete fields, Algebra i Analiz 9(1997) 98-147. 